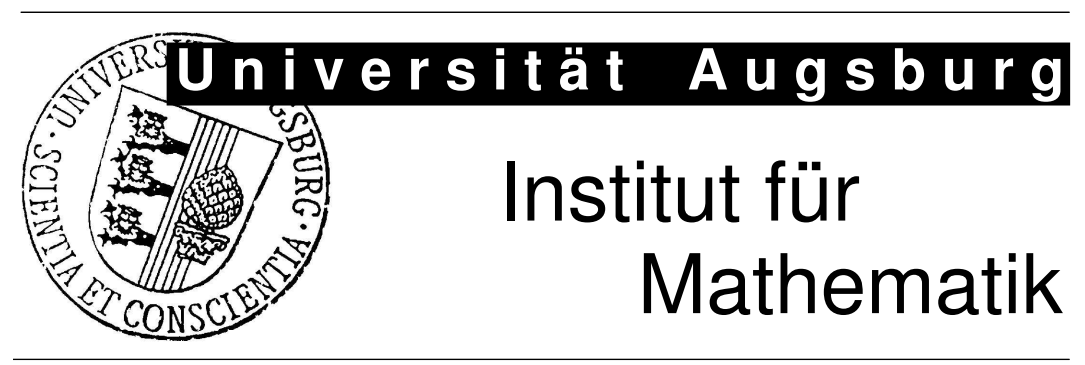

Ronald H.W. Hoppe, Zhiheng Liu

Snapshot Location by Error Equilibration in Proper Orthogonal Decomposition for Linear and Semilinear Parabolic Partial Differential Equations 


\section{Impressum:}

\section{Herausgeber:}

Institut für Mathematik

Universität Augsburg

86135 Augsburg

http://www . math. uni-augsburg.de/de/forschung/preprints.html

\section{ViSdP:}

Ronald H.W. Hoppe

Institut für Mathematik

Universität Augsburg

86135 Augsburg

Preprint: Sämtliche Rechte verbleiben den Autoren (C) 2013 


\title{
SNAPSHOT LOCATION BY ERROR EQUILIBRATION IN PROPER ORTHOGONAL DECOMPOSITION FOR LINEAR AND SEMILINEAR PARABOLIC PARTIAL DIFFERENTIAL EQUATIONS
}

\author{
R.H.W. HOPPE* AND Z. LIU ${ }^{\dagger}$
}

\begin{abstract}
It is well-known that the performance of snapshot based POD and POD-DEIM for spatially semidiscretized parabolic PDEs depends on the proper selection of the snapshot locations. In this contribution, we present an approach that for a fixed number of snapshots selects the location based on error equilibration in the sense that the global discretization error is approximately the same in each associated subinterval. The global discretization error is assessed by a hierarchical-type a posteriori error estimator known from automatic time-stepping for systems of ODEs. We study the impact of this snapshot selection on error equilibration for the ROM and provide numerical examples that illustrate the performance of the suggested approach.
\end{abstract}

Key words. POD, POD-DEIM, snapshot location, equilibration of the error, linear and semilinear parabolic PDEs

\section{AMS subject classifications.}

1. Introduction. During the past decades, there has been significant progress in the development, analysis, and efficient implementation of Model Order Reduction (MOR) for the numerical simulation of dynamical systems (systems of ordinary differential equations (ODEs)) and partial differential equations (PDEs). Among the most common techniques are Balanced Truncation Model Reduction (BTMR), Proper Orthogonal Decomposition (POD), and the Reduced Basis Method (RBM). The common aim is to construct a Reduced Order Model (ROM) of a significantly reduced dimension which essentially captures the dynamics of the original Full Order Model (FOM). This requires the specification of a ROM basis using data extracted from the FOM. In particular, one is interested both in a preservation of input-output relations and in a sufficiently accurate approximation of the state. For an overview of the stateof-the-art we refer to $[1,3,4,13,14,24,26,27,30,34]$ and the references therein. POD can be applied to both linear and nonlinear parabolic PDEs and is usually based on snapshots of the solution at selected time instants (snapshot locations). The snapshots can be chosen in function space (POD-Galerkin) or in Euclidean space for the spatially discretized PDEs. The basis for the ROM can be computed either by SVD of the matrix formed by the snapshots or by an Eigenvalue Decomposition (EVD) of associated covariance matrices. Estimates of the POD-Galerkin error in the state have been provided in $[15,20,21]$. As far as estimates of the POD state error in the ODE setting are concerned, we refer to the survey article [17] and the references therein.

For nonlinear dynamical systems, POD suffers from the fact that the computational complexity for the evaluation of the nonlinear mapping still depends on the dimension $N$ of the FOM. In [7], the authors have adopted the 'Empirical Interpolation Method' (EIM) from [2] to the discrete case ('Discrete Empirical Interpolation

\footnotetext{
*Institute of Mathematics, University of Augsburg, D-86159, Augsburg, Germany, and Department of Mathematics, University of Houston, Houston, TX 77204-3008, USA (rohop@math.uh.edu). The author has been supported by the DFG Priority Programs SPP 1253 and SPP 1506, by the NSF grants DMS-0914788, DMS-1115658, and by the European Science Foundation within the Networking Programme 'OPTPDE'.

${ }^{\dagger}$ Department of Mathematics, University of Houston, Houston, TX 77204-3008, USA. The author has been supported by the NSF grant DMS-1115658.
} 
Method' (DEIM)) and suggested an approach where the nonlinear function is approximated by a function whose evaluation is independent on $N$ and features a computational complexity being proportional to the number of reduced variables. This approach is now commonly referred to as POD-DEIM. Estimates of the POD-DEIM error in the state for spatially discretized nonlinear parabolic PDEs have been derived in $[8]$.

It is well-known that the performance of POD depends on a proper selection of the snapshot locations. Given a fixed number of snapshot locations, in [22] the authors have addressed the optimal selection of a certain number of additional snapshot locations. They have formulated this problem as a nonlinear optimization problem and have provided an SQP (Sequential Quadratic Programming)-type algorithm based on the second order sufficient optimality conditions. In this paper, we will focus on a different approach based on an equilibration of the POD or POD-DEIM error in the state. To be more precise, the snapshot locations are chosen in such a way that the state error is approximately the same in each subinterval of the resulting partition of the global time interval. The evaluation of the state error is realized by an efficient and reliable hierarchical a posteriori error estimator known from automatic time-stepping for systems of ODEs, namely the Euclidean norm of the difference of the solutions obtained by the implicit Euler scheme and the implicit trapezoidal rule. It turns out that the equilibration of the error is inherited by the POD or POD-DEIM based ROM. In the nonlinear case, this can be explained by means of the affine covariant version of the Newton-Kantorovich convergence theorem [11] for Newton's method which is used as an iterative scheme to compute the solutions of the nonlinear algebraic systems to be solved at each time step.

The paper is organized as follows: Section 2 deals with initial-boundary value problems for linear and semilinear second order parabolic PDEs and their semidiscretization by the finite element method. The semidiscretized problems are considered as the Full Order Models (FOMs). Section 3 gives a brief overview on snapshot based POD and POD-DEIM, whereas section 4 is devoted to snapshot selection by equilibration of the error. In the nonlinear case, implicit time stepping requires the solution of nonlinear algebraic systems which can be done by Newton's method. We perform a convergence analysis in an affine covariant framework with emphasis on the relationship between the Newton's method for the FOM and the POD and POD-DEIM based ROMs (subsections 4.1 and 4.2). In subsection 4.3, we provide conditions which guarantee that the error equilibration for the FOM is inherited by the respective ROMs. Finally, section 5 contains a detailed documentation of numerical results illustrating the performance of the suggested approach.

2. Linear and semilinear Parabolic PDEs and their semidiscretization in space. We use standard notation from Lebesgue and Sobolev space theory [31] and consider semilinear parabolic PDEs that can be written as abstract evolution equations according to

$$
\begin{aligned}
y^{\prime}(t)-A y(t)+f(t, y(t)) & =0, \quad t \in(0, T], \\
y(0) & =y_{0},
\end{aligned}
$$

in $L^{2}(\Omega)$, where $y^{\prime}(t):=d y(t) / d t$ and $A$ is a linear second order elliptic differential operator with $D(A)=H^{2}(\Omega) \cap H_{0}^{1}(\Omega), \Omega$ being a bounded polyhedral domain in $\mathbb{R}^{d}, d \in \mathbb{N}$, with boundary $\Gamma:=\partial \Omega$. We further assume $T>0, y_{0} \in L^{2}(\Omega)$, and $f \in C\left([0, T], L^{2}(\Omega)\right)$ with $f(t, 0)=0, t \in[0, T]$, satisfying a Lipschitz condition in the 
second argument, i.e.,

$$
\left\|f\left(t, y_{1}\right)-f\left(t, y_{2}\right)\right\|_{L^{2}(\Omega)} \leq L_{f}\left\|y_{1}-y_{2}\right\|_{L^{2}(\Omega)}, \quad y_{1}, y_{2} \in L^{2}(\Omega), t \in[0, T],
$$

for some constant $L_{f}>0$. In particular, we assume $A$ to be of the form

$$
A y:=\sum_{i, j=1}^{d} \frac{\partial}{\partial x_{j}}\left(a_{i j} \frac{\partial y}{\partial x_{i}}\right)-\sum_{i=1}^{d} b_{i} \frac{\partial y}{\partial x_{i}}-c y
$$

where $a_{i j} \in L^{\infty}(\Omega), 1 \leq i, j \leq d$, such that for some $\alpha>0$

$$
\sum_{i, j=1}^{d} a_{i j}(x) \xi_{i} \xi_{j} \geq \alpha|\xi|^{2}, \quad \xi \in \mathbb{R}^{d}, \text { f.a.a. } x \in \Omega,
$$

$b_{i} \in L^{\infty}(\Omega), 1 \leq i \leq d$, and $c \in L^{\infty}(\Omega)$ such that $c(x) \geq 0$ f.a.a. $x \in \Omega$. Moreover, we assume the coefficient functions to be such that $A-\omega I$ is dissipative for some $\omega>0$ with $D(A) \subset R(I-h A), 0<h<1 / \omega$. Then, the solution operator of $(2.1 \mathrm{a}),(2.1 \mathrm{~b})$ is a nonlinear semigroup $[18,19]$. In case $f$ does not depend on $y,(2.1 \mathrm{a}),(2.1 \mathrm{~b})$ represents a linear evolution equation whose solution operator is a strongly continuous linear semigroup.

Although snapshots may be considered in function space as in Galerkin-POD, the practical computation of snapshots is done with respect to a semidiscretization of the semilinear or linear evolution equation $(2.1 \mathrm{a}),(2.1 \mathrm{~b})$ in space, e.g., by the finite element method $[6,10]$. The finite element method is based on the variational formulation of (2.1a),(2.1b). We define function spaces

$$
\begin{aligned}
& W(0, T):=H^{1}\left((0, T), H^{-1}(\Omega)\right) \cap L^{2}\left((0, T), H_{0}^{1}(\Omega)\right), \\
& \bar{W}(0, T):=\left\{y \in W(0, T) \mid f(t, y) \in L^{2}(\Omega), t \in(0, T)\right\},
\end{aligned}
$$

and note that $W(0, T) \subset C\left([0, T], L^{2}(\Omega)\right)$ (cf., e.g., [29]). The variational formulation of $(2.1 \mathrm{a}),(2.1 \mathrm{~b})$ amounts to the computation of $y \in \bar{W}(0, T)$ such that for almost all $t \in(0, T)$ and $v \in V:=H_{0}^{1}(\Omega)$ it holds

$$
\begin{aligned}
&\left\langle\frac{\partial y}{\partial t}, v\right\rangle_{H^{-1}(\Omega), H_{0}^{1}(\Omega)}+a(y, v)+(f(y), v)_{L^{2}(\Omega)}=(g, v)_{L^{2}(\Omega)}, \\
&(y(0), v)_{L^{2}(\Omega)}=\left(y_{0}, v\right)_{L^{2}(\Omega)}
\end{aligned}
$$

where the bilinear form $a(\cdot, \cdot): H_{0}^{1}(\Omega) \times H_{0}^{1}(\Omega)$ is given by

$$
a(v, w):=\sum_{i, j=1}^{d} \int_{\Omega} a_{i j} \frac{\partial y}{\partial x_{i}} \frac{\partial y}{\partial x_{j}} d x+\sum_{i=1}^{d} \int_{\Omega} b_{i} \frac{\partial y}{\partial x_{i}} v d x+\int_{\Omega} c y v d x
$$

A function $y \in \bar{W}(0, T)$ satisfying (2.5a),(2.5b) is called a weak solution of (2.1a), (2.1b). For the existence of a weak solution we refer to [16] and [23]. In particular, under the previous assumptions on the operator $A$ and the nonlinear mapping $f$, the existence of a weak solution is guaranteed.

For the finite element approximation of $(2.5 \mathrm{a}),(2.5 \mathrm{~b})$ let $\left\{\mathcal{T}_{h}(\Omega)\right\}$ be a shape regular family of geometrically conforming simplicial triangulations of $\Omega$. We denote by $\mathcal{N}_{h}(\Omega)$ 
the set of nodal points of $\mathcal{T}_{h}(\Omega)$ in $\Omega$ and set $N:=\operatorname{card}\left(\mathcal{N}_{h}(\Omega)\right)$. For $T \in \mathcal{T}_{h}(\Omega)$ we refer to $h_{T}$ as the diameter of $T$ and set $h:=\max \left\{h_{T} \mid T \in \mathcal{T}_{h}(\Omega)\right\}$. We further refer to $V_{h} \subset V=H_{0}^{1}(\Omega)$ the finite element space of continuous, piecewise linear finite elements with respect to $\mathcal{T}_{h}(\Omega)$, i.e., denoting by $P_{1}(T)$ the set of polynomials of degree $\leq 1$ on $T$, we have

$$
V_{h}:=\left\{v_{h} \in C(\bar{\Omega})\left|v_{h}\right|_{T} \in P_{1}(T), T \in \mathcal{T}_{h}(\Omega),\left.v_{h}\right|_{\Gamma}=0\right\} .
$$

We note that $\operatorname{dim} V_{h}=N$ and $V_{h}=\operatorname{span}\left\{\varphi_{h}^{(1)}, \cdots, \varphi_{h}^{(N)}\right\}$, where $\varphi_{h}^{(i)}, 1 \leq i \leq N$, stands for the nodal basis function associated with the nodal point $a_{i} \in \mathcal{N}_{h}(\Omega)$. Then, the finite element approximation of $(2.5 \mathrm{a}),(2.5 \mathrm{~b})$ reads: Find $y_{h} \in C^{1}\left([0, T], V_{h}\right)$ such that for all $t \in[0, T]$ and $v_{h} \in V_{h}$ it holds

$$
\begin{aligned}
\left(y_{h}^{\prime}(t), v_{h}\right)_{L^{2}(\Omega)}+a\left(y_{h}, v_{h}\right)+( & \left.f\left(y_{h}\right), v_{h}\right)_{L^{2}(\Omega)}=\left(g, v_{h}\right)_{L^{2}(\Omega)}, \\
\left(y_{h}(0), v_{h}\right)_{L^{2}(\Omega)} & =\left(y_{0}, v_{h}\right)_{L^{2}(\Omega)} .
\end{aligned}
$$

For a priori estimates of the discretization error $y-y_{h}$ in term of the mesh width $h$ we refer to [32].

We may identify the finite element function $y_{h}(\cdot, t)$ with a vector $\mathbf{y}(t):=\left(y_{h, 1}(t)\right.$, $\left.\cdots, y_{h, N}(t)\right)^{T} \in \mathbb{R}^{N}$ such that $y_{h, i}(t):=y_{h}\left(a_{h, i}, t\right), a_{h, i} \in \mathcal{N}_{h}(\Omega), 1 \leq i \leq N$. We denote by $\mathbf{M} \in \mathbb{R}^{N \times N}$ and $\mathbf{A} \in \mathcal{R}^{N \times N}$ the mass and the stiffness matrix

$$
\begin{aligned}
(\mathbf{M})_{i j} & :=\left(\varphi_{h}^{(i)}, \varphi_{h}^{(j)}\right)_{L^{2}(\Omega)}, \quad 1 \leq i, j \leq N, \\
(\mathbf{A})_{i j} & :=a\left(\varphi_{h}^{(j)}, \varphi_{h}^{(i)}\right), \quad 1 \leq i, j \leq N .
\end{aligned}
$$

We further refer to $\mathbf{y}_{0} \in \mathbb{R}^{N}$ and $\mathbf{g} \in \mathbb{R}^{N}$ as the vectors with components

$$
\mathbf{y}_{i}:=\left(y_{0}, \varphi_{h}^{(i)}\right)_{L^{2}(\Omega)}, \quad, \mathbf{g}_{i}:=\left(g, \varphi_{h}^{(i)}\right)_{L^{2}(\Omega)}, \quad 1 \leq i \leq N,
$$

and to $\mathbf{f}(t, \mathbf{y}): \mathbb{R}^{N} \rightarrow \mathbb{R}^{N}, t \in[0, T]$, as the nonlinear map

$$
\begin{aligned}
& \mathbf{f}(t, \mathbf{y})=\left(f_{h, 1}\left(t, y_{h}\right), \cdots, f_{h, N}\left(t, y_{h}\right)\right)^{T} \\
& f_{h, i}\left(t, y_{h}\right):=\left(f\left(t, \sum_{j=1}^{N} y_{h, j} \varphi_{h}^{(j)}\right), \varphi_{h}^{(i)}\right)_{L^{2}(\Omega)}, \quad 1 \leq i \leq N .
\end{aligned}
$$

Then, the finite element approximation (2.8a), (2.8a) can be equivalently written as the following initial-value problem for a system of nonlinear first order ODEs

$$
\begin{aligned}
\mathbf{M y}^{\prime}(t)+\mathbf{A y}(t)+\mathbf{f}(t, \mathbf{y}(t)) & =0, \quad 0 \leq t \leq T, \\
\mathbf{M y}(0) & =\mathbf{y}_{0} .
\end{aligned}
$$

Since $\mathbf{f}$ is continuous and satisfies a Lipschitz condition in the second argument, due to the Theorem of Picard-Lindelöf the initial-value problem (2.9a),(2.9a) admits a unique solution.

In the sequel, we consider $(2.9 \mathrm{a}),(2.9 \mathrm{a})$ as the Full Order Model (FOM) for which we will describe the application of snapshot based POD and POD-DEIM in the following section 3 . 


\section{Snapshot Based POD and POD-DEIM.}

3.1. Snapshot Based POD. The method of snapshots for systems of ODEs assumes a certain number of snapshot times $t_{j}, 0 \leq j \leq M$, and snapshots $\mathbf{y}_{j} \in \mathbb{R}^{N}$ of the solution of $(2.9 \mathrm{a}),(2.9 \mathrm{~b})$. The aim is to find a set $\left\{\boldsymbol{\psi}_{i}\right\}_{i=1}^{n}, n \ll N$, of orthonormal vectors $\psi_{i} \in \mathbb{R}^{N}, 1 \leq i \leq n$, such that

$$
\begin{aligned}
& \min _{\left\{\boldsymbol{\psi}_{i}\right\}_{i=1}^{n}} J\left(\boldsymbol{\psi}_{1}, \cdots, \boldsymbol{\psi}_{n}\right):=\sum_{j=1}^{M}\left\|\mathbf{y}_{j}-\sum_{i=1}^{n}\left\langle\mathbf{y}_{j}, \boldsymbol{\psi}_{i}\right\rangle \boldsymbol{\psi}_{i}\right\|^{2}, \\
& \text { subject to }\left\langle\boldsymbol{\psi}_{i}, \boldsymbol{\psi}_{j}\right\rangle=\delta_{i j}, \quad 1 \leq i, j \leq n .
\end{aligned}
$$

The following well-known result establishes the necessary and sufficient optimality conditions for (3.1a), (3.1b).

Theorem 3.1. Let $\mathbf{K}:=\left[\mathbf{y}_{1}|\cdots| \mathbf{y}_{M}\right] \in \mathbb{R}^{N \times M}$. The necessary and sufficient optimality conditions for the minimization problem (3.1a),(3.1b) amount to the solution of the symmetric $N \times N$ eigenvalue problem

$$
\mathbf{K K}^{T} \boldsymbol{\psi}_{i}=\lambda_{i} \boldsymbol{\psi}_{i}, \quad 1 \leq i \leq n
$$

Proof. We refer to [33].

The orthonormal vectors $\psi_{i}, 1 \leq i \leq n$, can be computed by a Singular Value Decomposition (SVD) for $\mathbf{K} \in \mathbb{R}^{\bar{N} \times M}$ which requires the computation of orthogonal matrices

$$
\begin{aligned}
\mathbf{V}_{\ell} & =\left[\mathbf{u}_{1}|\cdots| \mathbf{u}_{N}\right] \in \mathbb{R}^{N \times N}, \\
\mathbf{V}_{r} & =\left[\mathbf{v}_{1}|\cdots| \mathbf{v}_{M}\right] \in \mathbb{R}^{M \times M},
\end{aligned}
$$

and a diagonal matrix

$$
\mathbf{D}=\operatorname{diag}\left(\sigma_{1}, \cdots, \sigma_{\ell}\right) \in \mathbb{R}^{\ell \times \ell},
$$

with $\sigma_{i}^{2}$ in decreasing order such that

$$
\begin{aligned}
& \mathbf{V}_{\ell}^{T} \mathbf{K} \mathbf{V}_{r}=\left(\begin{array}{cc}
\mathbf{D} & \mathbf{0} \\
\mathbf{0} & \mathbf{0}
\end{array}\right)=: \mathbf{\Sigma} \in \mathbb{R}^{N \times M}, \\
& \mathbf{K}_{i}=\sigma_{i} \mathbf{u}_{i}, \quad \mathbf{K}^{T} \mathbf{u}_{i}=\sigma_{i} \mathbf{v}_{i}, \quad \mathbf{K K}^{T} \mathbf{u}_{i}=\sigma_{i}^{2} \mathbf{u}_{i}, \quad 1 \leq i \leq \ell .
\end{aligned}
$$

The orthonormal POD basis $\left\{\psi_{i}\right\}_{i=1}^{n}, n \leq \ell$, and the associated eigenvalues $\lambda_{i}$ of $\mathbf{K K}^{T}$ are then given by

$$
\boldsymbol{\psi}_{i}=\mathbf{u}_{i}, \quad \lambda_{i}=\sigma_{i}^{2}>0, \quad 1 \leq i \leq n .
$$

The SVD for $K \in \mathbb{R}^{N \times n}$ implies the following error estimate.

Theorem 3.2. Let $\psi_{i}, 1 \leq i \leq n$, as in (3.5) and $\lambda_{i}=\sigma_{i}^{2}, 1 \leq i \leq \ell$, with $\sigma_{i}$ from (3.3c). Then it holds

$$
J\left(\psi_{1}, \cdots, \psi_{n}\right)=\sum_{j=1}^{M}\left\|\mathbf{y}_{j}-\sum_{i=1}^{n}\left\langle\mathbf{y}_{j}, \psi_{i}\right\rangle \psi_{i}\right\|^{2} \leq \sum_{i=n+1}^{\ell} \lambda_{i} .
$$


Proof. We refer to [33].

Setting $\mathbf{V}:=\left[\boldsymbol{\psi}_{1}|\cdots| \boldsymbol{\psi}_{n}\right] \in \mathbb{R}^{N \times n}$, the POD based ROM takes the form

$$
\begin{aligned}
\hat{\mathbf{M}} \hat{\mathbf{y}}^{\prime}(t)+\hat{\mathbf{A}} \hat{y}(t)+\hat{\mathbf{f}}(t, \hat{\mathbf{y}}(t)) & =0, \quad 0 \leq t \leq T \\
\hat{\mathbf{M}} \hat{\mathbf{y}}(0) & =\hat{\mathbf{y}}_{0}
\end{aligned}
$$

where $\hat{\mathbf{y}}_{0}:=V^{T} \mathbf{y}_{0}$, the matrices $\hat{\mathbf{M}}, \hat{\mathbf{A}} \in \mathbb{R}^{n \times n}$ are given by

$$
\hat{\mathbf{M}}:=\mathbf{V}^{T} \mathbf{M V}, \quad \hat{\mathbf{A}}:=\mathbf{V}^{T} \mathbf{A V},
$$

and the nonlinear function $\hat{\mathbf{f}}(t, \hat{\mathbf{y}}(t))$ reads as follows

$$
\hat{\mathbf{f}}(t, \hat{\mathbf{y}}(t)):=\mathbf{V}^{T} \mathbf{f}(t, \mathbf{V} \hat{\mathbf{y}}(t)), \quad 0 \leq t \leq T .
$$

REMARK 3.1. Denoting by $\mathbf{I}_{n}$ and $\mathbf{I}_{N}$ the $n \times n$ and $N \times N$ unit matrix, we have $\mathbf{V}^{T} \mathbf{V}=\mathbf{I}_{n}$. Moreover, for $\mathbf{x} \in \mathbb{R}^{N}$, the quantity $\tau_{P O D}(\mathbf{x}):=\left\|\left(\mathbf{I}_{N}-\mathbf{V V}^{T}\right) \mathbf{x}\right\|$ represents the optimal POD norm error which satisfies

$$
\sum_{m=1}^{M}\left\|\left(\mathbf{I}_{N}-\mathbf{V} \mathbf{V}^{T}\right) \mathbf{y}^{m}\right\|^{2} \leq \sum_{m=M+1}^{N} \lambda_{m}
$$

REMARK 3.2. We note that $\hat{\mathbf{f}}$ inherits the Lipschitz continuity in the second argument from $\mathbf{f}$ such that the reduced order initial-value problem (3.7a),(3.7b) is uniquely solvable.

REMARK 3.3. A commonly used criterion (cf., e.g., [25]) for the determination of the dimension $n$ of the ROM (3.7a),(3.7b) is to select some $\Theta \in(0,1)$ and to choose $n$ as the smallest integer for which

$$
\left(\sum_{i=1}^{n} \lambda_{i}\right) /\left(\sum_{i=1}^{\ell} \lambda_{i}\right) \geq \Theta
$$

For a discussion of this criterion and some more refined criteria we refer to [15].

3.2. POD-DEIM. POD-DEIM relies on two orthonormal matrices. The first one is the matrix $\mathbf{V} \in \mathbb{R}^{N \times n}$ obtained from the SVD of the snapshot matrix, whereas the second one is the matrix $\mathbf{W} \in \mathbb{R}^{N \times L}, L \ll N$, from the SVD of the nonlinear snapshot matrix $\mathcal{F} \mathcal{F}^{T}$, where $\mathcal{F}:=\left(\mathbf{f}\left(t_{1}, \mathbf{y}^{1}\right)|\cdots| \mathbf{f}\left(t_{L}, \mathbf{y}^{L}\right)\right)$. The DEIM is applied to the nonlinear function by using interpolation projection onto the columns of $\mathbf{W}$. To this end, one selects $L$ rows $p_{i}, 1 \leq i \leq L$, of $W$ and solves the linear algebraic system

$$
\left(\mathbf{P}^{T} \mathbf{W}\right) \mathbf{c}(t)=\mathbf{P}^{T} \mathbf{f}(t, \mathbf{V} \hat{\mathbf{y}}), \quad \mathbf{P}:=\left(\mathbf{e}_{p_{1}}|\cdots| \mathbf{e}_{p_{L}}\right) \in \mathbb{R}^{N \times L},
$$

where $\mathbf{e}_{i}$ stands for the i-th unit vector in $\mathbb{R}^{N}$. This results in the approximation

$$
\overline{\mathbf{f}}:=\mathbf{W}\left(\mathbf{P}^{T} \mathbf{W}\right)^{-1} \mathbf{P}^{T} \mathbf{f}
$$


of the nonlinear mapping $\mathbf{f}$. We note that the evaluation of $\mathbf{f}(t, \mathbf{V} \hat{\mathbf{y}})$ only requires $O(L)$ components of $\mathbf{V} \hat{\mathbf{y}}$.

As has been shown in [7], the DEIM error estimate is

$$
\|\mathbf{f}-\overline{\mathbf{f}}\| \leq\left\|\left(\mathbf{P}^{T} \mathbf{W}\right)^{-1}\right\|\left\|\left(\mathbf{I}_{N}-\mathbf{W} \mathbf{W}^{T}\right) \mathbf{f}\right\| \leq C_{D}\left\|\left(\mathbf{I}_{N}-\mathbf{W} \mathbf{W}^{T}\right) \mathbf{f}\right\| .
$$

Here

$$
C_{D}:=\left\|\left(\mathbf{P}^{T} \mathbf{W}\right)^{-1}\right\| \leq(1+\sqrt{2 N})^{L},
$$

and $\left\|\left(\mathbf{I}_{N}-\mathbf{W} \mathbf{W}^{T}\right) \mathbf{f}\right\|$ stands for the optimal POD-DEIM norm error which satisfies

$$
\sum_{\ell=1}^{L}\left\|\left(\mathbf{I}_{N}-\mathbf{W} \mathbf{W}^{T}\right) \mathbf{f}\left(t_{\ell}, \mathbf{y}^{\ell}\right)\right\|^{2} \leq \sum_{\ell=L+1}^{N} s_{\ell},
$$

where $s_{\ell}, 1 \leq \ell \leq N$, are the eigenvalues of the nonlinear snapshot matrix $\mathcal{F F}^{T}$ in decreasing order.

We define $\hat{\mathbf{f}}:[0, T] \times \mathbb{R}^{n} \rightarrow \mathbb{R}^{n}$ according to

$$
\hat{\mathbf{f}}(t, \hat{\mathbf{x}}):=\mathbf{V}^{T} \overline{\mathbf{f}}(t, \mathbf{V} \hat{\mathbf{x}}), \quad \hat{\mathbf{x}} \in \mathbb{R}^{n} .
$$

The POD-DEIM based ROM reads

$$
\begin{aligned}
\hat{\mathbf{M}} \hat{\mathbf{y}}^{\prime}(t)+\hat{\mathbf{A}} \hat{y}(t)+\hat{\mathbf{f}}(t, \hat{\mathbf{y}}(t)) & =0, \quad 0 \leq t \leq T, \\
\hat{\mathbf{M}} \hat{\mathbf{y}}(0) & =\hat{\mathbf{y}}_{0},
\end{aligned}
$$

4. Snapshot selection by Equilibration of the Error. Prescribing a fixed number $M+1$ of snapshots, we want to determine the snapshot locations $0=: t_{0}<$ $t_{1}<\cdots<t_{M}:=T$ in such a way that the global discretization error $\mathbf{y}\left(t_{m}\right)-\mathbf{y}^{m}$ is equidistributed, i.e., $\left\|\mathbf{y}\left(t_{m+1}\right)-\mathbf{y}^{m+1}\right\| \approx\left\|\mathbf{y}\left(t_{m}\right)-\mathbf{y}^{m}\right\|$ for all $1 \leq m \leq M-1$. Since the exact solution $y$ is not known, we substitute $\left\|\mathbf{y}\left(t_{m}\right)-\mathbf{y}^{m}\right\|$ by an efficient and reliable a posteriori error estimator $\eta_{m}$ and aim at $\eta^{m+1} \approx \eta^{m}, 1 \leq m \leq M-1$. Such a posteriori error estimators are well-known from automatic time-stepping for systems of ODEs (cf., e.g., [12]). Here, we consider a hierarchical-type estimator based on the difference between the solution by the implicit Euler scheme

$$
\begin{aligned}
\left(\mathbf{M}+\tau_{m} \mathbf{A}\right) \mathbf{y}^{m}+\tau_{m} \mathbf{f}\left(t_{m}, \mathbf{y}^{m}\right) & =\mathbf{M y}^{m-1}, \quad 1 \leq m \leq M, \\
\mathbf{M y}^{0} & =\mathbf{y}_{0},
\end{aligned}
$$

and the implicit trapezoidal rule

$$
\begin{aligned}
& \left(\mathbf{M}+\frac{\tau_{m}}{2} \mathbf{A}\right) \mathbf{y}_{T}^{m}+\frac{\tau_{m}}{2} \mathbf{f}\left(t_{m}, \mathbf{y}_{T}^{m}\right)= \\
& \left(\mathbf{M}-\frac{\tau_{m}}{2} \mathbf{A}\right) \mathbf{y}^{m-1}-\frac{\tau_{m}}{2} \mathbf{f}\left(t_{m-1}, \mathbf{y}^{m-1}\right), \quad 1 \leq m \leq M \\
& \mathbf{M y}_{T}^{0}=\mathbf{y}_{0},
\end{aligned}
$$

which is known to be convergent of order 2 provided $\mathbf{y} \in C^{3}([0, T])$. Hence, for sufficiently smooth $\mathbf{y}$ and sufficiently small $\tau$ the so-called saturation assumption

$$
\left\|\mathbf{y}\left(t_{m}\right)-\mathbf{y}_{T}^{m}\right\| \leq \theta\left\|\mathbf{y}\left(t_{m}\right)-\mathbf{y}^{m}\right\|, \quad 1 \leq m \leq M, 0 \leq \theta<1,
$$


can be expected to hold true which infers efficiency and reliability of the estimator $\eta_{m}:=\left\|\mathbf{y}^{m}-\mathbf{y}_{T}^{m}\right\|$ in the sense that

$$
\frac{1}{1+\theta} \eta_{m} \leq\left\|\mathbf{y}\left(t_{m}\right)-\mathbf{y}^{m}\right\| \leq \frac{1}{1-\theta} \eta_{m}
$$

In the sequel, we use a slightly modified estimator $\eta_{m}$. Rearranging terms in $(4.2 \mathrm{a})$, we get

$$
\begin{aligned}
& \left(\mathbf{M}+\tau_{m} \mathbf{A}\right) \mathbf{y}_{T}^{m}+\tau_{m} \mathbf{f}\left(t_{m}, \mathbf{y}_{T}^{m}\right)= \\
& \mathbf{M y}^{m-1}+\frac{\tau_{m}}{2} \mathbf{A}\left(\mathbf{y}_{T}^{m}-\mathbf{y}^{m-1}\right)-\frac{\tau_{m}}{2}\left(\mathbf{f}\left(t_{m-1}, \mathbf{y}^{m-1}\right)-\mathbf{f}\left(t_{m}, \mathbf{y}_{T}^{m}\right)\right)
\end{aligned}
$$

We replace $\mathbf{y}_{T}^{m}$ on the right-hand side of (4.5) with $\mathbf{y}^{m}$ and thus obtain

$$
\begin{aligned}
& \left(\mathbf{M}+\tau_{m} \mathbf{A}\right) \mathbf{y}_{T}^{m}+\tau_{m} \mathbf{f}\left(t_{m}, \mathbf{y}_{T}^{m}\right)= \\
& \mathbf{M y}^{m-1}+\frac{\tau_{m}}{2} \mathbf{A}\left(\mathbf{y}^{m}-\mathbf{y}^{m-1}\right)-\frac{\tau_{m}}{2}\left(\mathbf{f}\left(t_{m-1}, \mathbf{y}^{m-1}\right)-\mathbf{f}\left(t_{m}, \mathbf{y}^{m}\right)\right) .
\end{aligned}
$$

Setting $\mathbf{e}^{m}:=\mathbf{y}^{m}-\mathbf{y}_{T}^{m}$, it follows from (4.1) and (4.5) that $\mathbf{e}^{m}$ can be computed as the solution of

$$
\begin{aligned}
\left(\mathbf{M}+\tau_{m} \mathbf{A}\right) \mathbf{e}^{m}-\tau_{m} \mathbf{f}\left(t_{m}, \mathbf{y}^{m}-\mathbf{e}^{m}\right) & =\mathbf{g}\left(t_{m}\right), \quad 1 \leq m \leq M \\
\mathbf{M} \mathbf{e}^{0} & =\mathbf{0}
\end{aligned}
$$

where $\mathbf{g}\left(t_{m}\right)$ is given by

$$
\begin{aligned}
\mathbf{g}\left(t_{m}\right):= & \left(\mathbf{M}+\frac{\tau_{m}}{2} \mathbf{A}\right)\left(\mathbf{y}^{m}-\mathbf{y}^{m-1}\right)+\tau_{m} \mathbf{A} \mathbf{y}^{m-1} \\
& +\frac{\tau_{m}}{2}\left(\mathbf{f}\left(t_{m-1}, \mathbf{y}^{m-1}\right)-\mathbf{f}\left(t_{m}, \mathbf{y}^{m}\right)\right)
\end{aligned}
$$

For error equilibration, we set $\eta_{m}:=\left\|\mathbf{y}\left(t_{m}\right)-\mathbf{y}^{m}\right\|, \eta_{\mathrm{av}}:=M^{-1} \sum_{m=0}^{M} \eta_{m}$, and determine $\left\{t_{1}, \cdots, t_{M-1}\right\}$ as the solution of the minimization problem

$$
\min _{\left\{t_{1}, \cdots, t_{M-1}\right\}} \frac{1}{2} \sum_{m=1}^{M}\left|\eta_{m}-\eta_{\mathrm{av}}\right|^{2},
$$

subject to the constraints

$$
0=: t_{0}<t_{1}<\cdots<t_{M-1}<t_{M}:=T .
$$

The minimization problem (4.8a),(4.8b) is solved by SQP (Sequential Quadratic Programming) with BFGS updates [5].

4.1. Newton's Method for the POD Based ROM. Given snapshot locations $0=: t_{0}<t_{1}<\cdots<t_{M}:=T$, snapshots $\mathbf{y}^{m}, 0 \leq m \leq M$, can be computed by applying the implicit Euler scheme (4.1a),(4.1b) to the FOM (2.9a),(2.9b). The solution of (4.1a) requires the computation of a zero $\mathbf{x}^{*}$ of the nonlinear map $\mathbf{F}: \mathbb{R}^{N} \rightarrow \mathbb{R}^{N}$ given by

$$
\mathbf{F}(\mathbf{x}):=\left(\mathbf{M}+\tau_{m} \mathbf{A}\right) \mathbf{x}+\tau_{m} \mathbf{f}\left(t_{m}, \mathbf{x}\right)-\mathbf{M} \mathbf{y}^{m-1}, \quad x \in \mathbb{R}^{N}
$$


We observe that $\mathbf{F}$ inherits Lipschitz continuity from $\mathbf{f}$ in the sense that

$$
\left\|\mathbf{F}\left(\mathbf{y}_{1}\right)-\mathbf{F}\left(\mathbf{y}_{2}\right)\right\| \leq L_{F}\left\|\mathbf{y}_{1}-\mathbf{y}_{2}\right\|, \quad \mathbf{y}_{i} \in \mathbb{R}^{N}, 1 \leq i \leq 2 .
$$

The Lipschitz constant $L_{F}$ reads

$$
L_{F}:=\left\|\mathbf{M}+\tau_{m} \mathbf{A}\right\|+\tau_{m} L_{f},
$$

where $L_{f}$ is from $(2.2)$.

We further note that $\mathbf{M}+\tau_{m} \mathbf{A}$ is invertible. Moreover, if $\mathbf{f}$ is continuously differentiable in its second argument and $\tau_{m}$ is small enough, it follows from the Banach perturbation lemma (cf., e.g., [28]) that $\mathbf{F}$ is continuously differentiable with regular Jacobian

$$
\mathbf{F}^{\prime}(x):=\mathbf{M}+\tau_{m} \mathbf{A}+\tau_{m} \mathbf{f}_{\mathbf{y}}\left(t_{m}, \mathbf{x}\right), \quad \mathbf{x} \in \mathbb{R}^{N} .
$$

Hence, we may solve $\mathbf{F}(\mathbf{x})=\mathbf{0}$ by Newton's method

$$
\begin{aligned}
\mathbf{F}^{\prime}\left(\mathbf{x}^{k}\right) \boldsymbol{\Delta} \mathbf{x}^{k} & =-\mathbf{F}\left(\mathbf{x}^{k}\right), \\
\mathbf{x}^{k+1} & =\mathbf{x}^{k}+\Delta \mathbf{x}^{k}, \quad k \geq 0,
\end{aligned}
$$

using $\mathbf{x}^{0}:=\mathbf{y}^{m-1}$ as an initial iterate. Since Newton's method is affine covariant, i.e., invariant with respect to transformations in the range space, the convergence analysis should be provided in an affine covariant setting. An affine covariant version of the Newton-Kantorovich convergence theorem has been provided in [11].

TheOREM 4.1. Let $\mathbf{F}: D \subset \mathbb{R}^{N} \rightarrow \mathbb{R}^{N}$ be continuously differentiable on $D$ with an invertible Jacobian $\mathbf{F}^{\prime}\left(x^{0}\right)$ for some initial guess $\mathbf{x}^{0} \in D$. Assume further that the following conditions hold true:

$$
\begin{aligned}
& \left\|\mathbf{F}^{\prime}\left(\mathbf{x}^{0}\right)^{-1} \mathbf{F}\left(\mathbf{x}^{0}\right)\right\| \leq \alpha_{m}, \\
& \left\|\mathbf{F}^{\prime}\left(\mathbf{x}^{0}\right)^{-1}\left(\mathbf{F}^{\prime}\left(\mathbf{y}_{1}\right)-\mathbf{F}^{\prime}\left(\mathbf{y}_{2}\right)\right)\right\| \leq \gamma_{m}\|\mathbf{y}-\mathbf{x}\|, \quad \mathbf{y}_{i} \in D, 1 \leq i \leq 2, \\
& h_{m}:=\alpha_{m} \gamma_{m}<\frac{1}{2} \\
& \bar{B}\left(\mathbf{x}^{0}, \rho_{m}\right) \subset D, \rho_{m}:=\frac{1-\sqrt{1-2 h_{m}}}{\gamma_{m}} .
\end{aligned}
$$

Then, for the sequence $\left\{\mathbf{x}^{k}\right\}_{\mathbb{N}_{0}}$ of Newton iterates there holds

(i) $\mathbf{F}^{\prime}(\mathbf{x})$ is invertible for all Newton iterates $\mathbf{x}=\mathbf{x}^{k}, k \in \mathbb{N}_{0}$,

(ii) The sequence $\left\{\mathbf{x}^{k}\right\}_{\mathbb{N}}$ of Newton iterates is well defined with $\mathbf{x}^{k} \in \bar{B}\left(\mathbf{x}^{0}, \rho_{m}\right)$, $k \in \mathbb{N}_{0}$, and $\mathbf{x}^{k} \rightarrow \mathbf{x}^{*} \in \bar{B}\left(\mathbf{x}^{0}, \rho_{m}\right)(k \rightarrow \infty)$, where $\mathbf{F}\left(\mathbf{x}^{*}\right)=\mathbf{0}$,

(iii) The convergence $\mathbf{x}^{k} \rightarrow \mathbf{x}^{*}(k \rightarrow \infty)$ is quadratic.

(iv) The solution $\mathbf{x}^{*}$ of $\mathbf{F}(\mathbf{x})=0$ is unique in

$$
\bar{B}\left(\mathbf{x}^{0}, \rho_{m}\right) \cup\left(D \cap B\left(\mathbf{x}^{0}, \bar{\rho}_{m}\right)\right) \quad, \quad \bar{\rho}_{m}:=\frac{1+\sqrt{1-2 h_{m}}}{\gamma_{m}} .
$$

Proof. We refer to the proof of Theorem 2.1 in [11].

REMARK 4.1. The previous result tells us that Newton's method is locally quadratically convergent, i.e., the initial iterate $\mathbf{x}^{0}$ has to be situated in a sufficiently small 
neighborhood of a zero $\mathbf{x}^{*}$ of $\mathbf{F}$, the so-called Kantorovich neighborhood. If the initial iterate $\mathbf{x}^{0}$ does not satisfy the assumptions of Theorem 4.1, convergence of Newton's method can be achieved by appropriate globalization techniques. One of these techniques is the damped Newton method combined with a so-called monotonicity test for which we refer to [11].

The POD based ROM (3.7a),(3.7b) can be solved by the implicit Euler scheme as well giving rise to the solution of a nonlinear algebraic system $\hat{\mathbf{F}}(\hat{\mathbf{x}})=\mathbf{0}$ where $\hat{\mathbf{F}}: \mathbb{R}^{n} \rightarrow \mathbb{R}^{n}$ is given by

$$
\hat{\mathbf{F}}(\hat{\mathbf{x}}):=\left(\hat{\mathbf{M}}+\tau_{m} \hat{\mathbf{A}}\right) \hat{\mathbf{x}}+\tau_{m} \hat{\mathbf{f}}\left(t_{m}, \hat{\mathbf{x}}\right)-\hat{\mathbf{M}} \hat{\mathbf{y}}^{m-1}, \quad \hat{\mathbf{x}} \in \mathbb{R}^{n}
$$

The following result shows that $\hat{\mathbf{F}}$ inherits essential properties of $\mathbf{F}$.

THEOREM 4.2. If $\mathbf{F}$ is continuously differentiable in $D \subset \mathbb{R}^{N}$ with Jacobian $\mathbf{F}^{\prime}(\mathbf{x}), \mathbf{x} \in D$, then $\hat{\mathbf{F}}$ is continuously differentiable in $\hat{D} \subset \mathbb{R}^{n}, \mathbf{V} \hat{D} \subset D$, with Jacobian $\hat{\mathbf{F}}^{\prime}(\hat{\mathbf{x}}), \hat{\mathbf{x}} \in \hat{D}$, given by

$$
\hat{\mathbf{F}}^{\prime}(\hat{\mathbf{x}})=\mathbf{V}^{T} \mathbf{F}^{\prime}(\mathbf{V} \hat{\mathbf{x}}) \mathbf{V}
$$

Let the Jacobian $\mathbf{F}^{\prime}(\mathbf{x}), \mathbf{x} \in D$, be regular and assume that $\mathbf{F}^{\prime}$ satisfies the affine covariant Lipschitz condition

$$
\left\|\mathbf{F}^{\prime}(\mathbf{x})^{-1}\left(\mathbf{F}^{\prime}\left(\mathbf{y}_{1}\right)-\mathbf{F}^{\prime}\left(\mathbf{y}_{2}\right)\right)\right\| \leq \gamma_{m}\left\|\mathbf{y}_{1}-\mathbf{y}_{2}\right\|, \quad \mathbf{y}_{i} \in D, 1 \leq i \leq 2, \gamma_{m}>0
$$

Let $\hat{\mathbf{F}}_{\mathbf{V}}^{\prime}(\mathbf{x}): \mathbb{R}^{n} \rightarrow \mathbb{R}^{n}$ be given by

$$
\hat{\mathbf{F}}_{\mathbf{V}}^{\prime}(\mathbf{x}):=\mathbf{V}^{T} \mathbf{F}^{\prime}(\mathbf{x}) \mathbf{V}, \quad \mathbf{x} \in D
$$

Then, $\mathbf{V}^{T} \mathbf{F}^{\prime}(\mathbf{x})^{-1} \mathbf{V}$ is an approximate inverse of $\hat{\mathbf{F}}_{\mathbf{V}}^{\prime}(\mathbf{x})$ in the sense that

$$
\mathbf{V}^{T} \mathbf{F}^{\prime}(\mathbf{x})^{-1} \mathbf{V}=\hat{\mathbf{F}}_{\mathbf{V}}^{\prime}(\mathbf{x})^{-1}\left(\mathbf{I}_{n}-\mathbf{D}_{n}\right)
$$

where

$$
\mathbf{D}_{n}:=\mathbf{I}_{n}-\left(\mathbf{V}^{T} \mathbf{F}^{\prime}(\mathbf{x})\left(\mathbf{I}_{N}-\mathbf{V} \mathbf{V}^{T}\right) \mathbf{F}^{\prime}(\mathbf{x})^{-1} \mathbf{V}\right) .
$$

In particular, if $\left\|\left(\mathbf{I}_{N}-\mathbf{V} \mathbf{V}^{T}\right) \mathbf{F}^{\prime}(\mathbf{x})^{-1} \mathbf{V}\right\|$ satisfies

$$
\left\|\left(\mathbf{I}_{N}-\mathbf{V} \mathbf{V}^{T}\right) \mathbf{F}^{\prime}(\mathbf{x})^{-1} \mathbf{V}\right\| \leq q /\left(\left\|\mathbf{V}^{T} \mathbf{F}^{\prime}(\mathbf{x})\right\|\right),
$$

for some $0 \leq q<1$, we have

$$
\left\|\hat{\mathbf{F}}_{\mathbf{V}}^{\prime}(\mathbf{x})^{-1}\right\| \leq \frac{1}{1-q}\left\|\mathbf{V}^{T} \mathbf{F}^{\prime}(\mathbf{x})^{-1} \mathbf{V}\right\|
$$

Moreover, assume that $\hat{\mathbf{x}} \in \hat{D}$ satisfies

$$
\|\mathbf{x}-\mathbf{V} \hat{\mathbf{x}}\|<1 / C_{F^{\prime}}, \quad C_{F^{\prime}}:=\frac{\gamma_{m}}{1-q}\left\|\mathbf{V}^{T} \mathbf{F}^{\prime}(\mathbf{x})^{-1}\right\|\left\|\mathbf{V}^{T} \mathbf{F}^{\prime}(\mathbf{x})\right\| .
$$

Then, $\hat{\mathbf{F}}^{\prime}$ is regular in $\hat{\mathbf{x}}$ and it holds

$$
\left\|\hat{\mathbf{F}}^{\prime}(\hat{\mathbf{x}})^{-1}\right\| \leq \frac{1}{1-q}\left\|\mathbf{V}^{T} \mathbf{F}^{\prime}(\mathbf{x})^{-1}\right\|\left(1-C_{F^{\prime}}\|\mathbf{x}-\mathbf{V} \hat{\mathbf{x}}\|\right) .
$$


Proof. For the Jacobian $\hat{\mathbf{F}}^{\prime}(\hat{\mathbf{x}}), \hat{\mathbf{x}} \in \mathbb{R}^{n}$, we obtain

$$
\hat{\mathbf{F}}^{\prime}(\hat{\mathbf{x}})=\mathbf{V}^{T}\left(\mathbf{M}-\tau_{m} \mathbf{A}\right) \mathbf{V}+\tau_{m} \mathbf{V}^{T} \mathbf{f}_{\mathbf{y}}\left(t_{m}, \mathbf{V} \hat{\mathbf{x}}\right) \mathbf{V}=\mathbf{V}^{T} \mathbf{F}^{\prime}(\mathbf{V} \hat{\mathbf{x}}) \mathbf{V} .
$$

If $\mathbf{F}^{\prime}(\mathbf{x})$ is regular, so is $\hat{\mathbf{F}}_{\mathbf{V}}^{\prime}(\mathbf{x})$. Using $\mathbf{V}^{T} \mathbf{V}=\mathbf{I}_{n}$ we have

$$
\begin{aligned}
& \hat{\mathbf{F}}_{\mathbf{V}}^{\prime}(\mathbf{x}) \mathbf{V}^{T} \mathbf{F}^{\prime}(\mathbf{x})^{-1} \mathbf{V}= \\
& \mathbf{V}^{T} \mathbf{F}^{\prime}(\mathbf{x}) \mathbf{V} \mathbf{V}^{T} \mathbf{F}^{\prime}(\mathbf{x})^{-1} \mathbf{V}=\mathbf{I}_{n}-\mathbf{V}^{T} \mathbf{F}^{\prime}(\mathbf{x})\left(\mathbf{I}_{N}-\mathbf{V} \mathbf{V}^{T}\right) \mathbf{F}^{\prime}(\mathbf{x})^{-1} \mathbf{V},
\end{aligned}
$$

which implies (4.22). Under the assumption (4.24), $\mathbf{I}_{n}-\mathbf{D}_{n}$ is invertible, whence

$$
\mathbf{F}_{\mathbf{V}}^{\prime}(\mathbf{x})^{-1}=\mathbf{V}^{T} \mathbf{F}^{\prime}(\mathbf{x})^{-1} \mathbf{V}\left(\mathbf{I}_{n}-\mathbf{D}_{n}\right)^{-1}
$$

The estimate (4.25) is a consequence of the Neumann lemma (cf., e.g., [28]). Observing (4.20), it follows that

$$
\begin{aligned}
& \left\|\hat{\mathbf{F}}_{\mathbf{V}}^{\prime}(\mathbf{x})-\hat{\mathbf{F}}^{\prime}(\hat{\mathbf{x}})\right\|=\left\|\mathbf{V}^{T}\left(\mathbf{F}^{\prime}(\mathbf{x})-\mathbf{F}^{\prime}(\mathbf{V} \hat{\mathbf{x}})\right) \mathbf{V}\right\| \\
& \leq\left\|\mathbf{V}^{T} \mathbf{F}^{\prime}(\mathbf{x})\right\|\left\|\mathbf{F}^{\prime}(\mathbf{x})^{-1}\left(\mathbf{F}^{\prime}(\mathbf{x})-\mathbf{F}^{\prime}(\mathbf{V} \hat{\mathbf{x}})\right)\right\| \leq \gamma_{m}\left\|\mathbf{V}^{T} \mathbf{F}^{\prime}(\mathbf{x})\right\|\|\mathbf{x}-\mathbf{V} \hat{\mathbf{x}}\| .
\end{aligned}
$$

Under the assumption (4.26), the regularity of $\hat{\mathbf{F}}^{\prime}(\hat{\mathbf{x}})$ and the estimate (4.27) follow from the Banach perturbation lemma (cf., e.g., [28]).

REMARK 4.2. The assumption (4.26) in Theorem 4.2 can be expected to hold true, if $\left\|\hat{\mathbf{x}}-\mathbf{V}^{T} \mathbf{x}\right\|$ is sufficiently small as can be seen easily from

$$
\|\mathbf{x}-\mathbf{V} \hat{\mathbf{x}}\| \leq\left\|\mathbf{x}-\mathbf{V V}^{T} \mathbf{x}\right\|+\left\|\hat{\mathbf{x}}-\mathbf{V}^{T} \mathbf{x}\right\|=\tau_{P O D}(\mathbf{x})+\left\|\hat{\mathbf{x}}-\mathbf{V}^{T} \mathbf{x}\right\| .
$$

We are now in a position to show that the convergence properties of Newton's method for the FOM are inherited by the POD based ROM.

TheOREM 4.3. Assume that for $\mathbf{F}: D \subset \mathbb{R}^{N} \rightarrow \mathbb{R}^{N}$ the assumptions of Theorem 4.1 hold true and that (4.24) holds true for $\mathbf{x}=\mathbf{x}^{0}$. Then, for $\hat{\mathbf{F}}: \hat{D} \subset \mathbb{R}^{n} \rightarrow \mathbb{R}^{n}$ it holds

$$
\begin{aligned}
& \left\|\hat{\mathbf{F}}^{\prime}\left(\hat{\mathbf{x}}^{0}\right)^{-1} \hat{\mathbf{F}}\left(\hat{\mathbf{x}}^{0}\right)\right\| \leq \hat{\alpha}_{m}, \\
& \left\|\hat{\mathbf{F}}^{\prime}\left(\hat{\mathbf{x}}^{0}\right)^{-1}\left(\hat{\mathbf{F}}^{\prime}(\hat{\mathbf{y}})-\hat{\mathbf{F}}^{\prime}(\hat{\mathbf{x}})\right)\right\| \leq \hat{\gamma}_{m}\|\hat{\mathbf{y}}-\hat{\mathbf{x}}\|, \quad \hat{\mathbf{x}}, \hat{\mathbf{y}} \in \hat{D} .
\end{aligned}
$$

Here, the constants $\hat{\alpha}_{m}, \hat{\gamma}_{m}$ are given by

$$
\begin{aligned}
& \hat{\alpha}_{m}:= \\
& \frac{1}{1-q}\left\|\mathbf{V}^{T} \mathbf{F}^{\prime}\left(\mathbf{x}^{0}\right)^{-1}\right\|\left\|\mathbf{V}^{T} \mathbf{F}^{\prime}\left(\mathbf{x}^{0}\right)\right\|\left(1-C_{F^{\prime}}\left\|\mathbf{x}^{0}-\mathbf{V} \hat{\mathbf{x}}^{0}\right\|\right)\left(\alpha_{m}+\varepsilon_{m}\right), \\
& \hat{\gamma}_{m}:=\frac{\gamma_{m}}{1-q}\left\|\mathbf{V}^{T} \mathbf{F}^{\prime}\left(x^{0}\right)^{-1}\right\|\left\|\mathbf{V}^{T} \mathbf{F}^{\prime}\left(\mathbf{x}^{0}\right)\right\|\left(1-C_{F^{\prime}}\left\|\mathbf{x}^{0}-\mathbf{V} \hat{\mathbf{x}}^{0}\right\|\right) .
\end{aligned}
$$

where $\varepsilon_{m}$ in $(4.30 \mathrm{c})$ reads as follows

$$
\begin{aligned}
\varepsilon_{m}:= & \left\|\mathbf{F}^{\prime}\left(\mathbf{x}^{0}\right)\right\|^{-1}\left(\left\|\mathbf{V}^{T} \mathbf{M}\right\|\left\|\mathbf{y}^{m-1}-\mathbf{V}^{m-1}\right\|+\right. \\
& \left.\left(\left\|\mathbf{V}^{T}\left(\mathbf{M}+\tau_{m} \mathbf{A}\right)\right\|+\tau_{m} L_{f}\left\|\mathbf{V}^{T}\right\|\right)\left\|\mathbf{x}^{0}-\mathbf{V} \hat{\mathbf{x}}^{0}\right\|\right) .
\end{aligned}
$$


Moreover, assume that

$$
\hat{h}_{m}:=\hat{\alpha}_{m} \hat{\gamma}_{m}<\frac{1}{2}, \quad \overline{\hat{B}}\left(\hat{\mathbf{x}}^{0}, \hat{\rho}_{m}\right) \subset \hat{D}, \hat{\rho}_{m}:=\frac{1-\sqrt{1-2 \hat{h}_{m}}}{\hat{\gamma}_{m}} .
$$

Then, the sequence $\left\{\hat{\mathbf{x}}^{k}\right\}_{\mathbb{N}_{0}}$ of Newton iterates

$$
\begin{aligned}
\hat{\mathbf{F}}^{\prime}\left(\hat{\mathbf{x}}^{k}\right) \boldsymbol{\Delta} \hat{\mathbf{x}}^{k} & =-\hat{\mathbf{F}}\left(\hat{\mathbf{x}}^{k}\right), \\
\hat{\mathbf{x}}^{k+1} & =\hat{\mathbf{x}}^{k}+\boldsymbol{\Delta} \hat{\mathbf{x}}^{k}
\end{aligned}
$$

is well defined, stays in $\bar{B}\left(\hat{\mathbf{x}}^{0}, \hat{\rho}_{m}\right)$, and converges quadratically to some $\hat{\mathbf{x}}^{*} \in \overline{\hat{B}}\left(\hat{\mathbf{x}}^{0}, \hat{\rho}_{m}\right)$ with $\hat{\mathbf{F}}\left(\hat{\mathbf{x}}^{*}\right)=\mathbf{0}$. The solution $\hat{\mathbf{x}}^{*}$ is unique in $\bar{B}\left(\hat{\mathbf{x}}^{0}, \hat{\rho}_{m}\right) \cup\left(\hat{D} \cap \hat{B}\left(\hat{\mathbf{x}}^{0}, \overline{\hat{\rho}}_{m}\right)\right)$, $\overline{\hat{\rho}}_{m}:=\left(1+\sqrt{1-2 \hat{h}_{m}}\right) / \hat{\gamma}_{m}$.

Proof. In view of (4.18) we have

$$
\begin{aligned}
& \hat{\mathbf{F}}^{\prime}\left(\hat{\mathbf{x}}^{0}\right)^{-1} \hat{\mathbf{F}}\left(\hat{\mathbf{x}}^{0}\right)= \\
& \hat{\mathbf{F}}^{\prime}\left(\hat{\mathbf{x}}^{0}\right)^{-1}\left(\mathbf{V}^{T}\left(\left(\mathbf{M}+\tau_{m} \mathbf{A}\right) \mathbf{V} \hat{\mathbf{x}}^{0}+\tau_{m} \mathbf{f}\left(t_{m}, \mathbf{V} \hat{\mathbf{x}}^{0}\right)-\mathbf{M} \mathbf{V} \hat{\mathbf{y}}^{m-1}\right)\right)= \\
& \hat{\mathbf{F}}^{\prime}\left(\hat{\mathbf{x}}^{0}\right)^{-1} \mathbf{V}^{T}\left(\left(\mathbf{M}+\tau_{m} \mathbf{A}\right) \mathbf{x}^{0}+\tau_{m} \mathbf{f}\left(t_{m}, \mathbf{x}^{0}\right)-\mathbf{M} \mathbf{y}^{m-1}+\mathbf{M} \mathbf{y}^{m-1}-\mathbf{M V} \hat{\mathbf{y}}^{m-1}\right)- \\
& \left(\left(\mathbf{M}+\tau_{m} \mathbf{A}\right)\left(\mathbf{x}^{0}-\mathbf{V} \hat{\mathbf{x}}^{0}\right)+\tau_{m}\left(\mathbf{f}\left(t_{m}, \mathbf{x}^{0}\right)-\mathbf{f}\left(t_{m}, \mathbf{V} \hat{\mathbf{x}}^{0}\right)\right)\right) .
\end{aligned}
$$

Under the assumption (4.26) for $\mathbf{x}=\mathbf{x}^{0}$ and $\hat{\mathbf{x}}=\hat{\mathbf{x}}^{0}$, by (4.14) and (4.27) the first term on the right-hand side of (4.32) can be estimated from above as follows:

$$
\begin{aligned}
& \left\|\hat{\mathbf{F}}^{\prime}\left(\hat{\mathbf{x}}^{0}\right)^{-1} \mathbf{V}^{T}\left(\left(\mathbf{M}+\tau_{m} \mathbf{A}\right) \mathbf{x}^{0}+\tau_{m} \mathbf{f}\left(t_{m}, \mathbf{x}^{0}\right)-\mathbf{M} \mathbf{y}^{m-1}+\mathbf{M}\left(\mathbf{y}^{m-1}-\mathbf{V} \hat{\mathbf{y}}^{m-1}\right)\right)\right\|= \\
& \left\|\hat{\mathbf{F}}^{\prime}\left(\hat{\mathbf{x}}^{0}\right)^{-1} \mathbf{V}^{T}\left(\mathbf{F}^{\prime}\left(\mathbf{x}^{0}\right) \mathbf{F}^{\prime}\left(\mathbf{x}^{0}\right)^{-1} \mathbf{F}\left(\mathbf{x}^{0}\right)+\mathbf{M}\left(\mathbf{y}^{m-1}-\mathbf{V} \hat{\mathbf{y}}^{m-1}\right)\right)\right\| \leq \\
& \left\|\hat{\mathbf{F}}^{\prime}\left(\hat{\mathbf{x}}^{0}\right)^{-1}\right\|\left(\alpha_{m}\left\|\mathbf{V}^{T} \mathbf{F}^{\prime}\left(\mathbf{x}^{0}\right)\right\|+\left\|\mathbf{V}^{T} \mathbf{M}\right\|\left\|\mathbf{y}^{m-1}-\mathbf{V} \hat{\mathbf{y}}^{m-1}\right\|\right) \leq \\
& \frac{1}{1-q}\left\|\mathbf{V}^{T} \mathbf{F}^{\prime}\left(\mathbf{x}^{0}\right)^{-1}\right\|\left\|\mathbf{V}^{T} \mathbf{F}^{\prime}\left(\mathbf{x}^{0}\right)\right\|\left(1-C_{F^{\prime}}\left\|\mathbf{x}^{0}-\mathbf{V} \hat{\mathbf{x}}^{0}\right\|\right) . \\
& \cdot\left(\alpha_{m}+\left\|\mathbf{V}^{T} \mathbf{F}^{\prime}\left(\mathbf{x}^{0}\right)\right\|^{-1}\left\|\mathbf{V}^{T} \mathbf{M}\right\|\left\|\mathbf{y}^{m-1}-\mathbf{V} \hat{\mathbf{y}}^{m-1}\right\|\right) .
\end{aligned}
$$

For the second term on the right-hand side it follows from (4.14), (4.25), and (4.27) that

$$
\begin{aligned}
& \left\|\hat{\mathbf{F}}^{\prime}\left(\hat{\mathbf{x}}^{0}\right)^{-1} \mathbf{V}^{T}\left(\left(\mathbf{M}+\tau_{m} \mathbf{A}\right)\left(\mathbf{x}^{0}-\mathbf{V} \hat{\mathbf{x}}^{0}\right)+\tau_{m}\left(\mathbf{f}\left(t_{m}, \mathbf{x}^{0}\right)-\mathbf{f}\left(t_{m}, \mathbf{V} \hat{\mathbf{x}}^{0}\right)\right)\right)\right\| \leq \\
& \frac{1}{1-q}\left\|\mathbf{V}^{T} \mathbf{F}^{\prime}\left(\mathbf{x}^{0}\right)^{-1}\right\|\left\|\mathbf{V}^{T} \mathbf{F}^{\prime}\left(\mathbf{x}^{0}\right)\right\|\left(1-C_{F^{\prime}}\left\|\mathbf{x}^{0}-\mathbf{V} \hat{\mathbf{x}}^{0}\right\|\right) . \\
& \cdot\left(\left\|\mathbf{V}^{T}\left(\mathbf{M}+\tau_{m} \mathbf{A}\right)\right\|+\tau_{m} L_{f}\left\|\mathbf{V}^{T}\right\|\right)\left\|\mathbf{x}^{0}-\mathbf{V} \hat{\mathbf{x}}^{0}\right\| .
\end{aligned}
$$

The assertion (4.30a) can be deduced by using the preceding two estimates in (4.32). On the other hand, observing (4.15), (4.25), and (4.27), we obtain

$$
\begin{aligned}
& \left\|\hat{\mathbf{F}}^{\prime}\left(\hat{\mathbf{x}}^{0}\right)^{-1}\left(\hat{\mathbf{F}}^{\prime}\left(\hat{\mathbf{y}}_{1}\right)-\hat{\mathbf{F}}^{\prime}\left(\hat{\mathbf{y}}_{2}\right)\right)\right\|=\left\|\hat{\mathbf{F}}^{\prime}\left(\hat{\mathbf{x}}^{0}\right)^{-1} \mathbf{V}^{T}\left(\mathbf{F}^{\prime}\left(\mathbf{V} \hat{\mathbf{y}}_{1}\right)-\mathbf{F}^{\prime}\left(\mathbf{V} \hat{\mathbf{y}}_{2}\right)\right) \mathbf{V}\right\| \\
& \leq \gamma_{m}\left\|\hat{\mathbf{F}}^{\prime}\left(\hat{\mathbf{x}}^{0}\right)^{-1} \mathbf{V}^{T} \mathbf{F}^{\prime}\left(\mathbf{x}^{0}\right)\right\|\left\|\hat{\mathbf{y}}_{1}-\hat{\mathbf{y}}_{2}\right\| \\
& \leq \frac{\gamma_{m}}{1-q}\left\|\mathbf{V}^{T} \mathbf{F}^{\prime}\left(\mathbf{x}^{0}\right)^{-1}\right\|\left\|\mathbf{V}^{T} \mathbf{F}^{\prime}\left(\mathbf{x}^{0}\right)\right\|\left(1-C_{F^{\prime}}\left\|\mathbf{x}^{0}-\mathbf{V} \hat{\mathbf{x}}^{0}\right\|\right)\left\|\hat{\mathbf{y}}_{1}-\hat{\mathbf{y}}_{2}\right\|,
\end{aligned}
$$

which is (4.30). The rest of the assertions is now a consequence of Theorem 4.1. 
4.2. Newton's Method for the POD-DEIM Based ROM. Throughout this subsection and in the sequel, we assume that the function $\mathbf{f}:[0, T] \times \mathbb{R}^{N} \rightarrow$ $\mathbb{R}^{N}$ is continuously differentiable in its second argument with invertible Jacobian $\mathbf{f}_{\mathbf{y}}\left(t, \mathbf{x}^{0}\right), t \in[0, T]$, for some $\mathbf{x}^{0} \in D \subset \mathbb{R}^{N}$ and that $\mathbf{f}_{\mathbf{y}}$ satisfies the affine invariant Lipschitz condition

$$
\left\|\mathbf{f}_{\mathbf{y}}\left(t, \mathbf{x}^{0}\right)^{-1}\left(\mathbf{f}_{\mathbf{y}}\left(t, \mathbf{y}_{1}\right)-\mathbf{f}_{\mathbf{y}}\left(t, \mathbf{y}_{2}\right)\right)\right\| \leq \gamma_{f}\left\|\mathbf{y}_{1}-\mathbf{y}_{2}\right\|, \quad \mathbf{y}_{i} \in D, 1 \leq i \leq 2,
$$

for some $\gamma_{f}>0$.

If we solve (2.9a), (2.9b) with $\overline{\mathbf{f}}$ instead of $\mathbf{f}$ by the implicit Euler scheme, at each time step we have to solve a nonlinear algebraic system $\overline{\mathbf{F}}(\mathbf{x})=\mathbf{0}$, where the nonlinear $\operatorname{map} \overline{\mathbf{F}}: \mathbb{R}^{N} \rightarrow \mathbb{R}^{N}$ is given by

$$
\overline{\mathbf{F}}(x):=\left(\mathbf{M}+\tau_{m} \mathbf{A}\right) \mathbf{x}+\tau_{m} \overline{\mathbf{f}}\left(t_{m}, \mathbf{x}\right)-\mathbf{M} \mathbf{y}^{m-1} .
$$

On the other hand, if we solve the POD-DEIM based ROM (3.18a),(3.18b) by the implicit Euler scheme, we have to solve a nonlinear system with the nonlinear map $\hat{\mathbf{F}}: \mathbb{R}^{n} \rightarrow \mathbb{R}^{n}$

$$
\hat{\mathbf{F}}(\hat{\mathbf{x}}):=\left(\hat{\mathbf{M}}+\tau_{m} \hat{\mathbf{A}}\right) \hat{\mathbf{x}}+\tau_{m} \mathbf{V}^{T} \overline{\mathbf{f}}\left(t_{m}, \mathbf{V} \hat{\mathbf{x}}\right)-\hat{\mathbf{M}} \hat{\mathbf{y}}^{m-1}
$$

As in the previous subsection, we are interested in the properties of $\hat{\mathbf{F}}$.

THEOREM 4.4. Under the assumptions of Theorem 4.2 the POD-DEIM nonlinear map $\hat{\mathbf{F}}$ is continuously differentiable in $\hat{D} \subset \mathbb{R}^{n}, \mathbf{V} \hat{D} \subset D$, with the Jacobian $\hat{\mathbf{F}}^{\prime}(\hat{\mathbf{x}}), \hat{\mathbf{x}} \in \hat{D}$, given by

$$
\hat{\mathbf{F}}^{\prime}(\hat{\mathbf{x}})=\mathbf{V}^{T}\left(\mathbf{M}+\tau_{m} \mathbf{A}+\tau_{m} \overline{\mathbf{f}}_{\mathbf{y}}\left(t_{m}, \mathbf{V} \hat{\mathbf{x}}\right)\right) \mathbf{V}
$$

If $\hat{\mathbf{x}} \in \hat{D}$ satisfies

$$
\begin{aligned}
& \|\mathbf{x}-\mathbf{V} \hat{\mathbf{x}}\| \leq 1 / C_{F^{\prime}}, \\
& C_{F^{\prime}}:=(1-q)^{-1} \tau_{m} \gamma_{f} C_{D}\left\|\mathbf{V}^{T} \mathbf{F}^{\prime}(\mathbf{x})^{-1}\right\|\left\|\mathbf{V}^{T}\right\|\left\|\left(\mathbf{I}_{N}-\mathbf{W} \mathbf{W}^{T}\right) \mathbf{f}_{\mathbf{y}}\left(t_{m}, \mathbf{x}\right)\right\|,
\end{aligned}
$$

then $\hat{\mathbf{F}}^{\prime}$ is regular in $\hat{\mathbf{x}}$ and it holds

$$
\left\|\hat{\mathbf{F}}^{\prime}(\hat{\mathbf{x}})^{-1}\right\| \leq \frac{1}{1-q}\left\|\mathbf{V}^{T} \mathbf{F}^{\prime}(\mathbf{x})^{-1}\right\|\left(1-C_{F^{\prime}}\|\mathbf{x}-\mathbf{V} \hat{\mathbf{x}}\|\right)
$$

Proof. The representation (4.36) of the Jacobian follows directly from the definition of $\hat{F}$ by (4.34). Recalling (4.35) and (2.2), we further obtain

$$
\begin{aligned}
& \left\|\hat{\mathbf{F}}^{\prime}(\hat{\mathbf{x}})-\mathbf{F}_{\mathbf{V}}^{\prime}(\mathbf{x})\right\|=\left\|\mathbf{V}^{T}\left(\overline{\mathbf{F}}^{\prime}(\mathbf{V} \hat{\mathbf{x}})-\mathbf{F}^{\prime}(\mathbf{x})\right) \mathbf{V}\right\| \\
& \leq \tau_{m}\left\|\mathbf{V}^{T}\right\|\left\|\mathbf{W}\left(\mathbf{P}^{T} \mathbf{W}\right)^{-1} \mathbf{P}^{T} \mathbf{f}_{\mathbf{y}}\left(t_{m}, \mathbf{x}\right) \mathbf{f}_{\mathbf{y}}\left(t_{m}, \mathbf{x}\right)^{-1}\left(\mathbf{f}_{\mathbf{y}}\left(t_{m}, \mathbf{V} \hat{\mathbf{x}}\right)-\mathbf{f}_{\mathbf{y}}\left(t_{m}, \mathbf{x}\right)\right)\right\| \\
& \leq \tau_{m} \gamma_{f} C_{D}\left\|\mathbf{V}^{T}\right\|\left\|\left(\mathbf{I}_{N}-\mathbf{W} \mathbf{W}^{T}\right) \mathbf{f}_{\mathbf{y}}\left(t_{m}, \mathbf{x}\right)\right\|\|\mathbf{x}-\mathbf{V} \hat{\mathbf{x}}\| .
\end{aligned}
$$

Under the assumption (4.37) the estimate (4.38) follows again from the Banach perturbation lemma. 
TheOREM 4.5. Suppose that $\mathbf{F}: D \subset \mathbb{R}^{N} \rightarrow \mathbb{R}^{N}$ satisfies the assumptions of Theorem 4.1 and that (4.24) holds true for $\mathbf{x}=\mathbf{x}^{0}$. Then, for $\hat{\mathbf{F}}: \hat{D} \subset \mathbb{R}^{n} \rightarrow \mathbb{R}^{n}$ we have

$$
\begin{aligned}
& \left\|\hat{\mathbf{F}}^{\prime}\left(\hat{\mathbf{x}}^{0}\right)^{-1} \hat{\mathbf{F}}\left(\hat{\mathbf{x}}^{0}\right)\right\| \leq \hat{\alpha}_{m}, \\
& \left\|\hat{\mathbf{F}}^{\prime}\left(\hat{\mathbf{x}}^{0}\right)^{-1}\left(\hat{\mathbf{F}}^{\prime}(\hat{\mathbf{y}})-\hat{\mathbf{F}}^{\prime}(\hat{\mathbf{x}})\right)\right\| \leq \hat{\gamma}_{m}\|\hat{\mathbf{y}}-\hat{\mathbf{x}}\|, \quad \hat{\mathbf{x}}, \hat{\mathbf{y}} \in \hat{D} .
\end{aligned}
$$

Here, the constants $\hat{\alpha}_{m}, \hat{\gamma}_{m}$ are given by

$$
\begin{aligned}
& \hat{\alpha}_{m}:= \\
& \frac{1}{1-q}\left\|\mathbf{V}^{T} \mathbf{F}^{\prime}\left(\mathbf{x}^{0}\right)^{-1}\right\|\left\|\mathbf{V}^{T} \mathbf{F}^{\prime}\left(\mathbf{x}^{0}\right)\right\|\left(1-C_{F^{\prime}}\left\|\mathbf{x}^{0}-\mathbf{V} \hat{\mathbf{x}}^{0}\right\|\right)\left(\alpha_{m}+\varepsilon_{m}^{(1)}\right), \\
& \hat{\gamma}_{m}:= \\
& \frac{1}{1-q}\left\|\mathbf{V}^{T} \mathbf{F}^{\prime}\left(\mathbf{x}^{0}\right)^{-1}\right\| \mathbf{V}^{T} \mathbf{F}^{\prime}\left(\mathbf{x}^{0}\right) \|\left(1-C_{F^{\prime}}\left\|\mathbf{x}^{0}-\mathbf{V} \hat{\mathbf{x}}^{0}\right\|\right)\left(\gamma_{m}+\varepsilon_{m}^{(2)}\right) .
\end{aligned}
$$

where $\varepsilon_{m}^{(i)}, 1 \leq i \leq 2$, read as follows

$$
\begin{aligned}
\varepsilon_{m}^{(1)}:= & \left\|\mathbf{V}^{T} \mathbf{F}^{\prime}\left(\mathbf{x}^{0}\right)\right\|^{-1}\left(\left(\left\|\mathbf{V}^{T}\left(\mathbf{M}+\tau_{m} \mathbf{A}\right)\right\|+\tau_{m} C_{D} L_{f}\left\|\mathbf{V}^{T}\right\|\right)\left\|\mathbf{x}^{0}-\mathbf{V} \hat{\mathbf{x}}^{0}\right\|+\right. \\
& \left.\tau_{m} C_{D}\left\|\mathbf{V}^{T}\right\|\left\|\left(\mathbf{I}_{N}-\mathbf{W} \mathbf{W}^{T}\right) \mathbf{f}\left(t_{m}, \mathbf{x}^{0}\right)\right\|+\left\|\mathbf{V}^{T} \mathbf{M}\right\|\left\|\mathbf{y}^{m-1}-\mathbf{V} \hat{\mathbf{y}}^{m-1}\right\|\right), \\
\varepsilon_{m}^{(2)}:= & \tau_{m} \gamma_{f} C_{D}\left\|\mathbf{V}^{T} \mathbf{F}^{\prime}\left(\mathbf{x}^{0}\right)\right\|^{-1}\left\|\mathbf{V}^{T}\right\|\left\|\left(\mathbf{I}_{N}-\mathbf{W} \mathbf{W}^{T}\right) \mathbf{f}_{\mathbf{y}}\left(t_{m}, \mathbf{x}^{0}\right)\right\| .
\end{aligned}
$$

Moreover, assume that

$$
\hat{h}_{m}:=\hat{\alpha}_{m} \hat{\gamma}_{m}<\frac{1}{2}, \quad \bar{B}\left(\hat{\mathbf{x}}^{0}, \hat{\rho}_{m}\right) \subset \hat{D}, \hat{\rho}_{m}:=\frac{1-\sqrt{1-2 \hat{h}_{m}}}{\hat{\gamma}_{m}} .
$$

Then, the sequence $\left\{\hat{\mathbf{x}}^{k}\right\}_{\mathbb{N}_{0}}$ of Newton iterates

$$
\begin{aligned}
\hat{\mathbf{F}}^{\prime}\left(\hat{\mathbf{x}}^{k}\right) \boldsymbol{\Delta} \hat{\mathbf{x}}^{k} & =-\hat{\mathbf{F}}\left(\hat{\mathbf{x}}^{k}\right), \\
\hat{\mathbf{x}}^{k+1} & =\hat{\mathbf{x}}^{k}+\boldsymbol{\Delta} \hat{\mathbf{x}}^{k}
\end{aligned}
$$

is well defined, stays in $\overline{\hat{B}}\left(\hat{\mathbf{x}}^{0}, \hat{\rho}_{m}\right)$, and converges quadratically to some $\hat{\mathbf{x}}^{*} \in \overline{\hat{B}}\left(\hat{\mathbf{x}}^{0}, \hat{\rho}_{m}\right)$ with $\hat{\mathbf{F}}\left(\hat{\mathbf{x}}^{*}\right)=\mathbf{0}$. The solution $\hat{\mathbf{x}}^{*}$ is unique in $\hat{\hat{B}}\left(\hat{\mathbf{x}}^{0}, \hat{\rho}_{m}\right) \cup\left(\hat{D} \cap \hat{B}\left(\hat{\mathbf{x}}^{0}, \overline{\hat{\rho}}_{m}\right)\right), \overline{\hat{\rho}}_{m}:=$ $\left(1+\sqrt{1-2 \hat{h}_{m}}\right) / \hat{\gamma}_{m}$.

Proof. Due to the definition of $\hat{\mathbf{F}}$ by (4.35) we have

$$
\begin{aligned}
& \hat{\mathbf{F}}^{\prime}\left(\hat{\mathbf{x}}^{0}\right)^{-1} \hat{\mathbf{F}}\left(\hat{\mathbf{x}}^{0}\right)= \\
& \hat{\mathbf{F}}^{\prime}\left(\hat{\mathbf{x}}^{0}\right)^{-1} \mathbf{V}^{T}\left(\left(\mathbf{M}+\tau_{m} \mathbf{A}\right) \mathbf{V} \hat{\mathbf{x}}^{0}+\tau_{m} \mathbf{W}\left(\mathbf{P}^{T} \mathbf{W}\right)^{-1} \mathbf{P}^{T} \mathbf{f}\left(t_{m}, \mathbf{V} \hat{\mathbf{x}}^{0}\right)-\mathbf{M} \mathbf{V} \hat{\mathbf{y}}^{m-1}\right)= \\
& \hat{\mathbf{F}}^{\prime}\left(\hat{\mathbf{x}}^{0}\right)^{-1} \mathbf{V}^{T}\left(\left(\mathbf{M}+\tau_{m} \mathbf{A}\right) \mathbf{x}^{0}+\tau_{m} \mathbf{f}\left(t_{m}, \mathbf{x}^{0}\right)-\mathbf{M y} \mathbf{y}^{m-1}+\mathbf{M}\left(\mathbf{y}^{m-1}-\mathbf{V} \hat{\mathbf{y}}^{m-1}\right)\right)- \\
& \mathbf{V}^{T}\left(\left(\mathbf{M}+\tau_{m} \mathbf{A}\right)\left(\mathbf{x}^{0}-\mathbf{V} \hat{\mathbf{x}}^{0}\right)+\tau_{m}\left(\mathbf{f}\left(t_{m}, \mathbf{x}^{0}\right)-\mathbf{W}\left(\mathbf{P}^{T} \mathbf{W}\right)^{-1} \mathbf{P}^{T} \mathbf{f}\left(t_{m}, \mathbf{x}^{0}\right)\right)\right)- \\
& \left.\tau_{m} \mathbf{V}^{T} \mathbf{W}\left(\mathbf{P}^{T} \mathbf{W}\right)^{-1} \mathbf{P}^{T}\left(\mathbf{f}\left(t_{m}, \mathbf{x}^{0}\right)-\mathbf{f}\left(t_{m}, \mathbf{V} \hat{\mathbf{x}}^{0}\right)\right)\right)=\hat{\mathbf{F}}^{\prime}\left(\hat{\mathbf{x}}^{0}\right)^{-1} \mathbf{V}^{T}\left(\mathbf{F}^{\prime}\left(\mathbf{x}^{0}\right) \mathbf{F}^{\prime}\left(\mathbf{x}^{0}\right)^{-1} \mathbf{F}\left(\mathbf{x}^{0}\right)\right. \\
& \left.+\mathbf{M}\left(\mathbf{y}^{m-1}-\mathbf{V} \hat{\mathbf{y}}^{m-1}\right)\right)-\mathbf{V}^{T}\left(\left(\mathbf{M}+\tau_{m} \mathbf{A}\right)\left(\mathbf{x}^{0}-\mathbf{V} \hat{\mathbf{x}}^{0}\right)+\tau_{m}\left(\mathbf{f}\left(t_{m}, \mathbf{x}^{0}\right)-\right.\right. \\
& \left.\left.\mathbf{W}\left(\mathbf{P}^{T} \mathbf{W}\right)^{-1} \mathbf{P}^{T} \mathbf{f}\left(t_{m}, \mathbf{x}^{0}\right)\right)\right)-\tau_{m} \mathbf{V}^{T} \mathbf{W}\left(\mathbf{P}^{T} \mathbf{W}\right)^{-1} \mathbf{P}^{T}\left(\mathbf{f}\left(t_{m}, \mathbf{x}^{0}\right)-\mathbf{f}\left(t_{m}, \mathbf{V} \hat{\mathbf{x}}^{0}\right)\right) .
\end{aligned}
$$


Under the assumption (4.26) for $\mathbf{x}=\mathbf{x}^{0}$ and $\hat{\mathbf{x}}=\hat{\mathbf{x}}^{0}$, by (4.14) and (4.27) the first term on the right-hand side of (4.42) can be estimated from above as follows:

$$
\begin{aligned}
& \left\|\hat{\mathbf{F}}^{\prime}\left(\hat{\mathbf{x}}^{0}\right)^{-1} \mathbf{V}^{T}\left(\mathbf{F}^{\prime}\left(\mathbf{x}^{0}\right) \mathbf{F}^{\prime}\left(\mathbf{x}^{0}\right)^{-1} \mathbf{F}\left(\mathbf{x}^{0}\right)+\mathbf{M}\left(\mathbf{y}^{m-1}-\mathbf{V} \hat{\mathbf{y}}^{m-1}\right)\right)\right\| \\
& \leq \frac{1}{1-q}\left\|\mathbf{V}^{T} \mathbf{F}^{\prime}\left(\mathbf{x}^{0}\right)^{-1}\right\|\left(1-C_{F^{\prime}}\left\|\mathbf{x}^{0}-\mathbf{V} \hat{\mathbf{x}}^{0}\right\|\right) . \\
& \quad \cdot\left(\left\|\alpha_{m}\right\| \mathbf{V}^{T} \mathbf{F}^{\prime}\left(\mathbf{x}^{0}\right)\|+\| \mathbf{V}^{T} \mathbf{M}\|\| \mathbf{y}^{m-1}-\mathbf{V} \hat{\mathbf{y}}^{m-1} \|\right) .
\end{aligned}
$$

Using (4.27) and (3.14), for the second term we obtain

$$
\begin{aligned}
& \left\|\hat{\mathbf{F}}^{\prime}\left(\hat{\mathbf{x}}^{0}\right)^{-1} \mathbf{V}^{T}\left(\left(\mathbf{M}+\tau_{m} \mathbf{A}\right)\left(\mathbf{x}^{0}-\mathbf{V} \hat{\mathbf{x}}^{0}\right)+\tau_{m}\left(\mathbf{f}\left(t_{m}, \mathbf{x}^{0}\right)-\mathbf{W}\left(\mathbf{P}^{T} \mathbf{W}\right)^{-1} \mathbf{P}^{T} \mathbf{f}\left(t_{m}, \mathbf{x}^{0}\right)\right)\right)\right\| \\
& \leq \frac{1}{1-q}\left\|\mathbf{V}^{T} \mathbf{F}^{\prime}\left(\mathbf{x}^{0}\right)^{-1}\right\|\left(1-C_{F^{\prime}}\left\|\mathbf{x}^{0}-\mathbf{V} \hat{\mathbf{x}}^{0}\right\|\right)\left(\left\|\mathbf{V}^{T}\left(\mathbf{M}+\tau_{m} \mathbf{A}\right)\right\|\left\|\mathbf{x}^{0}-\mathbf{V} \hat{\mathbf{x}}^{0}\right\|+\right. \\
& \left.\quad \tau_{m} C_{D}\left\|\mathbf{V}^{T}\right\|\left\|\left(\mathbf{I}_{N}-\mathbf{W} \mathbf{W}^{T}\right) \mathbf{f}\left(t_{m}, \mathbf{x}^{0}\right)\right\|\right) .
\end{aligned}
$$

Finally, observing (2.2), (4.27), and (3.15), the third can be estimated from above according to

$$
\begin{aligned}
& \left\|\hat{\mathbf{F}}^{\prime}\left(\hat{\mathbf{x}}^{0}\right)^{-1} \mathbf{V}^{T}\left(\tau_{m} \mathbf{V}^{T} \mathbf{W}\left(\mathbf{P}^{T} \mathbf{W}\right)^{-1} \mathbf{P}^{T}\left(\mathbf{f}\left(t_{m}, \mathbf{x}^{0}\right)-\mathbf{f}\left(t_{m}, \mathbf{V} \hat{\mathbf{x}}^{0}\right)\right)\right)\right\| \\
& \leq \frac{1}{1-q} \tau_{m} C_{D} L_{f}\left\|\mathbf{V}^{T} \mathbf{F}^{\prime}\left(\mathbf{x}^{0}\right)^{-1}\right\|\left\|\mathbf{V}^{T}\right\|\left(1-C_{F^{\prime}}\left\|\mathbf{x}^{0}-\mathbf{V} \hat{\mathbf{x}}^{0}\right\|\right)\left\|\mathbf{x}^{0}-\mathbf{V} \hat{\mathbf{x}}^{0}\right\| .
\end{aligned}
$$

Using the preceding three estimates in (4.42), it follows that

$$
\begin{aligned}
& \left\|\hat{\mathbf{F}}^{\prime}\left(\hat{\mathbf{x}}^{0}\right)^{-1} \hat{\mathbf{F}}\left(\hat{\mathbf{x}}^{0}\right)\right\| \\
& \leq \frac{1}{1-q}\left\|\mathbf{V}^{T} \mathbf{F}^{\prime}\left(\mathbf{x}^{0}\right)^{-1}\right\|\left(1-C_{F^{\prime}}\left\|\mathbf{x}^{0}-\mathbf{V} \hat{\mathbf{x}}^{0}\right\|\right)\left(\alpha_{m}\left\|\mathbf{V}^{T} \mathbf{F}^{\prime}\left(\mathbf{x}^{0}\right)\right\|+\right. \\
& \left(\left\|\mathbf{V}^{T}\left(\mathbf{M}+\tau_{m} \mathbf{A}\right)\right\|+\tau_{m} C_{D} L_{f}\left\|\mathbf{V}^{T}\right\|\right)\left\|\mathbf{x}^{0}-\mathbf{V} \hat{\mathbf{x}}^{0}\right\|+\left\|\mathbf{V}^{T} \mathbf{M}\right\|\left\|\mathbf{y}^{m}-\mathbf{V} \hat{\mathbf{y}}^{m-1}\right\|+ \\
& \left.\tau_{m} C_{D}\left\|\mathbf{V}^{T}\right\|\left\|\left(\mathbf{I}_{N}-\mathbf{W} \mathbf{W}^{T}\right) \mathbf{f}\left(t_{m}, \mathbf{x}^{0}\right)\right\|\right)
\end{aligned}
$$

which readily gives (4.40a). In order to prove (4.40b), observing (4.35) we find

$$
\begin{aligned}
& \hat{\mathbf{F}}^{\prime}\left(\hat{\mathbf{x}}^{0}\right)^{-1}\left(\hat{\mathbf{F}}^{\prime}\left(\hat{\mathbf{y}}_{1}\right)-\hat{\mathbf{F}}^{\prime}\left(\hat{\mathbf{y}}_{2}\right)\right)= \\
& \hat{\mathbf{F}}^{\prime}\left(\hat{\mathbf{x}}^{0}\right)^{-1}\left(\mathbf{V}^{T}\left(\mathbf{M}+\tau_{m} \mathbf{A}\right) \mathbf{V}\left(\hat{\mathbf{y}}_{1}-\hat{\mathbf{y}}_{2}\right)+\tau_{m} \mathbf{V}^{T} \mathbf{W}\left(\mathbf{P}^{T} \mathbf{W}\right)^{-1} \mathbf{P}^{T}\left(\mathbf{f}_{\mathbf{y}}\left(t_{m}, \mathbf{V} \hat{\mathbf{y}}_{1}\right)-\right.\right. \\
& \left.\left.\mathbf{f}_{\mathbf{y}}\left(t_{m}, \mathbf{V} \hat{\mathbf{y}}_{1}\right)\right) \mathbf{V}\right)=\hat{\mathbf{F}}^{\prime}\left(\hat{\mathbf{x}}^{0}\right)^{-1}\left(\mathbf{V}^{T}\left(\mathbf{M}+\tau_{m} \mathbf{A}\right) \mathbf{V}\left(\hat{\mathbf{y}}_{1}-\hat{\mathbf{y}}_{2}\right)+\tau_{m} \mathbf{V}^{T}\left(\mathbf{f}_{\mathbf{y}}\left(t_{m}, \mathbf{V} \hat{\mathbf{y}}_{1}\right)-\right.\right. \\
& \left.\tau_{m} \mathbf{f}_{\mathbf{y}}\left(t_{m}, \mathbf{V} \hat{\mathbf{y}}_{1}\right)\right)\left(\mathbf{V}^{T}\left(\mathbf{I}_{N}-\mathbf{W}\left(\mathbf{P}^{T} \mathbf{W}\right)^{-1} \mathbf{P}^{T}\right)\left(\mathbf{f}_{\mathbf{y}}\left(t_{m}, \mathbf{V} \hat{\mathbf{y}}_{1}\right)-\mathbf{f}_{\mathbf{y}}\left(t_{m}, \mathbf{V} \hat{\mathbf{y}}_{1}\right)\right) \mathbf{V}\right)= \\
& \left.\mathbf{f}_{\mathbf{y}}\left(t_{m}, \mathbf{V} \hat{\mathbf{y}}_{2}\right)\right) \mathbf{V} \hat{\mathbf{F}}^{\prime}\left(\hat{\mathbf{x}}^{0}\right)^{-1}\left(\mathbf{V}^{T}\left(\mathbf{F}^{\prime}\left(\mathbf{V} \hat{\mathbf{y}}_{1}\right)-\mathbf{F}^{\prime}\left(\mathbf{V} \hat{\mathbf{y}}_{2}\right)\right) \mathbf{V}-\right. \\
& \left.\tau_{m} \mathbf{V}^{T}\left(\mathbf{I}_{N}-\mathbf{W}\left(\mathbf{P}^{T} \mathbf{W}\right)^{-1} \mathbf{P}^{T}\right)\left(\mathbf{f}_{\mathbf{y}}\left(t_{m}, \mathbf{V} \hat{\mathbf{y}}_{1}\right)-\mathbf{f}_{\mathbf{y}}\left(t_{m}, \mathbf{V} \hat{\mathbf{y}}_{2}\right)\right) \mathbf{V}\right) .
\end{aligned}
$$

Using (4.15) and (4.38), for the first term on the right-hand side of (4.44) we obtain 
the estimate

$$
\begin{aligned}
& \left\|\hat{\mathbf{F}}^{\prime}\left(\hat{x}^{0}\right)^{-1} \mathbf{V}^{T}\left(\mathbf{F}^{\prime}\left(\mathbf{V} \hat{\mathbf{y}}_{1}\right)-\mathbf{F}^{\prime}\left(\mathbf{V} \hat{\mathbf{y}}_{2}\right)\right) \mathbf{V}\right\|= \\
& \left\|\hat{\mathbf{F}}^{\prime}\left(\hat{x}^{0}\right)^{-1} \mathbf{V}^{T} \mathbf{F}^{\prime}\left(\mathbf{x}^{0}\right) \mathbf{F}^{\prime}\left(\mathbf{x}^{0}\right)^{-1}\left(\mathbf{F}^{\prime}\left(\mathbf{V} \hat{\mathbf{y}}_{1}\right)-\mathbf{F}^{\prime}\left(\mathbf{V} \hat{\mathbf{y}}_{2}\right)\right) \mathbf{V}\right\| \leq \\
& \frac{\gamma_{m}}{1-q}\left\|\mathbf{V}^{T} \mathbf{F}^{\prime}\left(\mathbf{x}^{0}\right)^{-1}\right\|\left\|\mathbf{V}^{T} \mathbf{F}^{\prime}\left(\mathbf{x}^{0}\right)\right\|\left(1-C_{F^{\prime}}\left\|\mathbf{x}^{0}-\mathbf{V} \hat{\mathbf{x}}^{0}\right\|\right)\left\|\hat{\mathbf{y}}_{1}-\hat{\mathbf{y}}_{2}\right\| .
\end{aligned}
$$

Taking advantage of (4.33), (4.38), and (3.14), the second term can be estimated from above according to

$$
\begin{aligned}
& \tau_{m}\left\|\hat{\mathbf{F}}^{\prime}\left(\hat{x}^{0}\right)^{-1} \mathbf{V}^{T}\left(\mathbf{I}_{N}-\mathbf{W}\left(\mathbf{P}^{T} \mathbf{W}\right)^{-1} \mathbf{P}^{T}\right)\left(\mathbf{f}_{\mathbf{y}}\left(t_{m}, \mathbf{V} \hat{\mathbf{y}}_{1}\right)-\mathbf{f}_{\mathbf{y}}\left(t_{m}, \mathbf{V} \hat{\mathbf{y}}_{2}\right)\right) \mathbf{V}\right\| \leq \\
& \frac{\tau_{m}}{1-q} C_{D}\left\|\mathbf{V}^{T} \mathbf{F}^{\prime}\left(\mathbf{x}^{0}\right)^{-1}\right\|\left\|\mathbf{V}^{T}\right\|\left(1-C_{F^{\prime}}\left\|\mathbf{x}^{0}-\mathbf{V} \hat{\mathbf{x}}^{0}\right\|\right)\left\|\left(\mathbf{I}_{N}-\mathbf{W} \mathbf{W}^{T}\right) \mathbf{f}_{\mathbf{y}}\left(t_{m}, \mathbf{x}^{0}\right)\right\| \cdot \\
& \cdot\left\|\mathbf{f}_{\mathbf{y}}\left(t_{m}, \mathbf{x}^{0}\right)^{-1}\left(\mathbf{f}_{\mathbf{y}}\left(t_{m}, \mathbf{V} \hat{\mathbf{y}}_{1}\right)-\mathbf{f}_{\mathbf{y}}\left(t_{m}, \mathbf{V} \hat{\mathbf{y}}_{2}\right)\right)\right\| \leq \\
& \tau_{m} \gamma_{f} C_{D}\left\|\mathbf{V}^{T} \mathbf{F}^{\prime}\left(\mathbf{x}^{0}\right)^{-1}\right\|\left\|\mathbf{V}^{T}\right\|\left(1-C_{F^{\prime}}\left\|\mathbf{x}^{0}-\mathbf{V} \hat{\mathbf{x}}^{0}\right\|\right) . \\
& \cdot\left\|\left(\mathbf{I}_{N}-\mathbf{W} \mathbf{W}^{T}\right) \mathbf{f}_{\mathbf{y}}\left(t_{m}, \mathbf{x}^{0}\right)\right\|\left\|\hat{\mathbf{y}}_{1}-\hat{\mathbf{y}}_{2}\right\| .
\end{aligned}
$$

Then, (4.40b) is a consequence of (4.44) and the preceding two estimates. The rest of the assertions follow from Theorem 4.1.

4.3. Error Equilibration for the POD and POD-DEIM Based ROMs. In this subsection, we show that the error equilibration for the FOM is inherited by the POD and POD-DEIM based ROMs. The proof relies on the affine covariant version of the Newton-Kantorovich theorem 4.1 applied to the respective ROMs and on the assessment of the POD and POD-DEIM error from [33] and [8].

We first study the POD based ROM. Recalling the equations (4.7a),(4.7b) satisfied by $\mathbf{e}^{m}:=\mathbf{y}^{m}-\mathbf{y}_{T}^{m}$, we note that $\mathbf{e}^{0}=\mathbf{0}$, whereas $\mathbf{e}^{m}, 1 \leq m \leq M$, is a zero of the nonlinear map $\mathbf{F}: \mathbb{R}^{N} \rightarrow \mathbb{R}^{N}$ given by

$$
\mathbf{F}(\mathbf{x}):=\left(\mathbf{M}+\tau_{m} \mathbf{A}\right) \mathbf{x}-\tau_{m} \mathbf{f}\left(t_{m}, \mathbf{y}^{m}-\mathbf{x}\right)-\mathbf{g}\left(t_{m}\right)
$$

with Jacobian

$$
\mathbf{F}^{\prime}(\mathbf{x}):=\left(\mathbf{M}+\tau_{m} \mathbf{A}\right)+\tau_{m} \mathbf{f}_{\mathbf{y}}\left(t_{m}, \mathbf{y}^{m}-\mathbf{x}\right) .
$$

We solve $\mathbf{F}(\mathbf{x})=\mathbf{0}$ by Newton's method with initial iterate $\mathbf{x}^{0}=\mathbf{0}$.

The POD based ROM requires the computation of $\hat{\mathbf{e}}^{m}, 1 \leq m \leq M$, as a zero of the nonlinear map $\hat{\mathbf{F}}: \mathbb{R}^{n} \rightarrow \mathbb{R}^{n}$ given by

$$
\hat{\mathbf{F}}(\hat{\mathbf{x}}):=\left(\hat{\mathbf{M}}+\tau_{m} \hat{\mathbf{A}}\right) \hat{\mathbf{x}}-\tau_{m} \mathbf{V}^{T} \mathbf{f}\left(t_{m}, \mathbf{y}^{m}-\mathbf{V} \hat{\mathbf{x}}\right)-\mathbf{V}^{T} \mathbf{g}\left(t_{m}\right)
$$

with Jacobian

$$
\hat{\mathbf{F}}^{\prime}(\hat{\mathbf{x}}):=\left(\hat{\mathbf{M}}+\tau_{m} \hat{\mathbf{A}}\right)+\tau_{m} \mathbf{V}^{T} \mathbf{f}_{\mathbf{y}}\left(t_{m}, \mathbf{y}^{m}-\mathbf{V} \hat{\mathbf{x}}\right) \mathbf{V}=\mathbf{V}^{T} \mathbf{F}^{\prime}(\mathbf{V} \hat{\mathbf{x}}) \mathbf{V}
$$

We compute $\hat{\mathbf{e}}^{m}$ by Newton's method with initial iterate $\hat{\mathbf{x}}^{0}=\mathbf{0}$. If we assume

$$
\left\|\left(\mathbf{I}_{N}-\mathbf{V} \mathbf{V}^{T}\right) \mathbf{F}^{\prime}(\mathbf{0})^{-1} \mathbf{V}\right\| \leq q /\left\|\mathbf{V}^{T} \mathbf{F}^{\prime}(\mathbf{0})\right\|
$$


for some $0 \leq q<1$ and take $\mathbf{F}^{\prime}(\mathbf{0})=\mathbf{V}^{T} \mathbf{F}^{\prime}(\mathbf{0}) \mathbf{V}$ into account, as in Theorem 4.2 we find that $\mathbf{F}^{\prime}(\mathbf{0})$ is regular and satisfies

$$
\left\|\mathbf{F}^{\prime}(\mathbf{0})^{-1}\right\| \leq \frac{1}{1-q}\left\|\mathbf{V}^{T} \mathbf{F}^{\prime}(\mathbf{0})^{-1}\right\| .
$$

Then, the affine covariant Newton Kantorovich theorem applied to the POD based ROM reads as follows:

TheOREM 4.6. Let $\mathbf{F}: D \subset \mathbb{R}^{N} \rightarrow \mathbb{R}^{N}$ fulfill the assumptions of Theorem 4.1 for $\mathbf{x}^{0}=\mathbf{0}$, i.e.,

$$
\begin{aligned}
& \left\|\mathbf{F}^{\prime}(\mathbf{0})^{-1} \mathbf{F}(\mathbf{0})\right\| \leq \alpha, \\
& \left\|\mathbf{F}^{\prime}(\mathbf{0})^{-1}\left(\mathbf{F}^{\prime}\left(\mathbf{y}_{1}\right)-\mathbf{F}^{\prime}\left(\mathbf{y}_{2}\right)\right)\right\| \leq \gamma\left\|\mathbf{y}_{1}-\mathbf{y}_{2}\right\|, \quad \mathbf{y}_{i} \in D, 1 \leq i \leq 2, \\
& h:=\alpha \gamma<\frac{1}{2}, \\
& \bar{B}(\mathbf{0}, \rho) \subset D, \rho:=\frac{1-\sqrt{1-2 h}}{\gamma} .
\end{aligned}
$$

Moreover, suppose that (4.49) is satisfied. Then, for $\hat{\mathbf{F}}: \hat{D} \subset \mathbb{R}^{n} \rightarrow \mathbb{R}^{n}$ it holds

$$
\begin{aligned}
& \left\|\hat{\mathbf{F}}^{\prime}(\mathbf{0})^{-1} \hat{\mathbf{F}}(\mathbf{0})\right\| \leq \hat{\alpha}, \\
& \left\|\hat{\mathbf{F}}^{\prime}(\mathbf{0})^{-1}\left(\hat{\mathbf{F}}^{\prime}\left(\hat{\mathbf{y}}_{1}\right)-\hat{\mathbf{F}}^{\prime}\left(\hat{\mathbf{y}}_{2}\right)\right)\right\| \leq \hat{\gamma}\left\|\hat{\mathbf{y}}_{1}-\hat{\mathbf{y}}_{2}\right\|, \quad \hat{\mathbf{y}}_{i} \in \hat{D}, 1 \leq i \leq 2,
\end{aligned}
$$

where the constants $\hat{\alpha}, \hat{\gamma}$ are given by

$$
\begin{aligned}
& \hat{\alpha}:=\frac{\alpha}{1-q}\left\|\mathbf{V}^{T} \mathbf{F}^{\prime}(\mathbf{0})^{-1}\right\|\left\|\mathbf{V}^{T} \mathbf{F}^{\prime}(\mathbf{0})\right\|, \\
& \hat{\gamma}:=\frac{\gamma}{1-q}\left\|\mathbf{V}^{T} \mathbf{F}^{\prime}(\mathbf{0})^{-1}\right\|\left\|\mathbf{V}^{T} \mathbf{F}^{\prime}(\mathbf{0})\right\| .
\end{aligned}
$$

Moreover, assume that

$$
\hat{h}:=\hat{\alpha} \hat{\gamma}<\frac{1}{2}, \quad \overline{\hat{B}}(\mathbf{0}, \hat{\rho}) \subset \hat{D}, \hat{\rho}:=\frac{1-\sqrt{1-2 \hat{h}}}{\hat{\gamma}} .
$$

Then, the sequence $\left\{\hat{\mathbf{x}}^{k}\right\}_{\mathbb{N}_{0}}$ of Newton iterates is well defined, stays in $\overline{\hat{B}}(\mathbf{0}, \hat{\rho})$, and converges quadratically to $\hat{\mathbf{e}}^{m} \in \overline{\hat{B}}(\mathbf{0}, \hat{\rho})$ with $\hat{\mathbf{F}}\left(\hat{\mathbf{e}}^{m}\right)=\mathbf{0}$. The solution $\hat{\mathbf{e}}^{m}$ is unique in $\overline{\hat{B}}(\mathbf{0}, \hat{\rho}) \cup(\hat{D} \cap \hat{B}(\mathbf{0}, \overline{\hat{\rho}})), \overline{\hat{\rho}}:=(1+\sqrt{1-2 \hat{h}}) / \hat{\gamma}$.

Proof. Using (4.50), we find

$$
\begin{aligned}
\left\|\hat{\mathbf{F}}^{\prime}(\mathbf{0})^{-1} \hat{\mathbf{F}}(\mathbf{0})\right\| & \leq\left\|\hat{\mathbf{F}}^{\prime}(\mathbf{0})^{-1}\right\|\left\|\mathbf{V}^{T} \mathbf{F}^{\prime}(\mathbf{0}) \mathbf{F}^{\prime}(\mathbf{0})^{-1} \mathbf{F}(\mathbf{0}) \mathbf{V}\right\| \\
& \leq \frac{\alpha}{1-q}\left\|\mathbf{V}^{T} \mathbf{F}^{\prime}(\mathbf{0})^{-1}\right\|\left\|\mathbf{V}^{T} \mathbf{F}^{\prime}(\mathbf{0})\right\|
\end{aligned}
$$

as well as

$$
\begin{aligned}
\| \hat{\mathbf{F}}^{\prime}(\mathbf{0})^{-1}\left(\hat{\mathbf{F}}^{\prime}(\hat{\mathbf{y}})-\hat{\mathbf{F}}^{\prime}(\hat{\mathbf{x}}) \|\right. & \leq\left\|\hat{\mathbf{F}}^{\prime}(\mathbf{0})^{-1}\right\|\left\|\mathbf{V}^{T} \mathbf{F}^{\prime}(\mathbf{0}) \mathbf{F}^{\prime}(\mathbf{0})^{-1}(\mathbf{F}(\mathbf{V} \hat{\mathbf{y}})-\mathbf{F}(\mathbf{V} \hat{\mathbf{x}})) \mathbf{V}\right\| \\
& \leq \frac{\gamma_{m}}{1-q}\left\|\mathbf{V}^{T} \mathbf{F}^{\prime}(\mathbf{0})^{-1}\right\|\left\|\mathbf{V}^{T} \mathbf{F}^{\prime}(\mathbf{0})\right\|\|\hat{\mathbf{y}}-\hat{\mathbf{x}}\| .
\end{aligned}
$$


The assertions follow from Theorem 4.1.

On the other hand, we know from [33] that there exists a constant $C>0$ such that

$$
\begin{aligned}
& \left\|\mathbf{e}^{m}-\mathbf{V} \hat{\mathbf{e}}^{m}\right\|=\| \mathbf{y}^{m}-\mathbf{y}_{T}^{m}-\left(\mathbf{V} \hat{\mathbf{y}}^{m}-\mathbf{V} \hat{\mathbf{y}}_{T}^{m} \| \leq\right. \\
& \left\|\mathbf{y}^{m}-\mathbf{V} \hat{\mathbf{y}}^{m}\right\|+\left\|\mathbf{y}_{T}^{m}-\mathbf{V} \hat{\mathbf{y}}_{T}^{m}\right\| \leq C \tau_{\mathrm{POD}}, \quad \tau_{\mathrm{POD}}:=\left(\sum_{\ell=n+1}^{N} \lambda_{\ell}\right)^{1 / 2} .
\end{aligned}
$$

Setting $\hat{\eta}_{m}:=\left\|\hat{\mathbf{e}}^{m}\right\|, 1 \leq m \leq M$, and combining the results of Theorem 4.6 and (4.54), we can prove error equilibration of the POD based ROM.

THEOREM 4.7. In addition to the assumptions of Theorem 4.6 suppose that $\delta_{m}:=C \tau_{P O D} / \eta_{m} \ll 1,1 \leq m \leq M$. Then, for $\eta_{m}=\eta\left(1-\varepsilon_{m}\right), \varepsilon_{m} \ll 1$, and $\Lambda:=\max (\hat{\rho}, \rho)$ it holds

$$
\Lambda^{-1}\left(1-\varepsilon_{m}\right)\left(1-\delta_{m}\right) \leq \frac{\hat{\eta}_{m}}{\hat{\eta}_{m-1}} \leq \Lambda\left(1-\delta_{m-1}\right)^{-1}\left(1-\varepsilon_{m-1}\right)^{-1}
$$

Proof. We have

$$
\left\|\mathbf{e}^{m}-\mathbf{V} \hat{\mathbf{e}}^{m}\right\| \geq\left\|\mathbf{e}^{m}\right\|-\left\|\mathbf{V} \hat{\mathbf{e}}^{m}\right\| \geq\left\|\mathbf{e}^{m}\right\|-\left\|\hat{\mathbf{e}}^{m}\right\|=\eta_{m}-\hat{\eta}_{m},
$$

and hence, in view of (4.54) it follows that

$$
\hat{\eta}_{m} \geq \eta_{m}-\left\|\mathbf{e}^{m}-\mathbf{V} \hat{\mathbf{e}}^{m}\right\| \geq \eta_{m}-C \tau_{\mathrm{POD}}=\eta_{m}\left(1-C \tau_{\mathrm{POD}} / \eta_{m}\right) .
$$

Theorem 4.6 tells us

$$
\hat{\eta}_{m} \leq \hat{\rho}, \quad 1 \leq m \leq M
$$

Consequently, combining (4.56) and (4.57) we find

$$
\frac{\hat{\eta}_{m}}{\hat{\eta}_{m-1}} \geq \frac{\eta_{m}\left(1-C \tau_{\mathrm{POD}} / \eta_{m}\right)}{\hat{\rho}} \geq \frac{\eta_{m}\left(1-C \tau_{\mathrm{POD}} / \eta_{m}\right)}{\max (\hat{\rho}, \rho)}
$$

and

$$
\frac{\hat{\eta}_{m-1}}{\hat{\eta}_{m}} \geq \frac{\eta_{m-1}\left(1-C \tau_{\mathrm{POD}} / \eta_{m-1}\right)}{\hat{\rho}} \geq \frac{\eta_{m-1}\left(1-C \tau_{\mathrm{POD}} / \eta_{m-1}\right)}{\max (\hat{\rho}, \rho)}
$$

This leads to

$$
\frac{\eta_{m}\left(1-C \tau_{\mathrm{POD}} / \eta_{m}\right)}{\max (\hat{\rho}, \rho)} \leq \frac{\hat{\eta}_{m}}{\hat{\eta}_{m-1}} \leq \frac{\max (\hat{\rho}, \rho)}{\eta_{m-1}\left(1-C \tau_{\mathrm{POD}} / \eta_{m-1}\right)},
$$

which readily results in $(4.56)$.

A similar result can be derived for the POD-DEIM based ROM. In this case, we have

$$
\hat{\mathbf{F}}(\hat{\mathbf{x}}):=\left(\hat{\mathbf{M}}-\tau_{m} \hat{\mathbf{A}}\right) \hat{\mathbf{x}}-\tau_{m} \mathbf{V}^{T} \overline{\mathbf{f}}\left(t_{m}, \mathbf{y}^{m}-\mathbf{V} \hat{\mathbf{x}}\right)-\mathbf{V}^{T} \mathbf{g}\left(t_{m}\right),
$$

with $\overline{\mathbf{f}}$ from (3.13). The Jacobian reads

$$
\hat{\mathbf{F}}^{\prime}(\hat{\mathbf{x}})=\hat{\mathbf{M}}-\tau_{m} \hat{\mathbf{A}}+\tau_{m} \mathbf{V}^{T} \overline{\mathbf{f}}_{\mathbf{y}}\left(t_{m}, \mathbf{y}^{m}-\mathbf{V} \hat{\mathbf{x}}\right) \mathbf{V} .
$$


THEOREM 4.8. Suppose that $\mathbf{F}: D \subset \mathbb{R}^{N} \rightarrow \mathbb{R}^{N}$ satisfies the assumptions of Theorem 4.6 and that (4.49) is satisfied. Then, for $\hat{\mathbf{F}}: \hat{D} \subset \mathbb{R}^{n} \rightarrow \mathbb{R}^{n}$ we have

$$
\begin{aligned}
& \left\|\hat{\mathbf{F}}^{\prime}(\mathbf{0})^{-1} \hat{\mathbf{F}}(\mathbf{0})\right\| \leq \hat{\alpha}_{m} \\
& \left\|\hat{\mathbf{F}}^{\prime}(\mathbf{0})^{-1}\left(\hat{\mathbf{F}}^{\prime}\left(\hat{\mathbf{y}}_{1}\right)-\hat{\mathbf{F}}^{\prime}\left(\hat{\mathbf{y}}_{2}\right)\right)\right\| \leq \hat{\gamma}_{m}\left\|\hat{\mathbf{y}}_{1}-\hat{\mathbf{y}}_{2}\right\|, \quad \hat{\mathbf{y}}_{i} \in \hat{D}, 1 \leq i \leq 2
\end{aligned}
$$

The constants $\hat{\alpha}_{m}, \hat{\gamma}_{m}$ read

$$
\begin{aligned}
\hat{\alpha}_{m} & :=\hat{\alpha}\left(1+\kappa_{m}^{(1)},\right. \\
\hat{\gamma}_{m} & :=\hat{\gamma}\left(1+\kappa_{m}^{(2)}\right),
\end{aligned}
$$

where $\hat{\alpha}, \hat{\gamma}$, and $\kappa_{m}^{(i)}, 1 \leq i \leq 2$, are given by

$$
\begin{aligned}
\hat{\alpha} & :=\frac{\alpha}{1-q}\left\|\mathbf{V}^{T} \mathbf{F}^{\prime}(\mathbf{0})^{-1}\right\|\left\|\mathbf{V}^{T} \mathbf{F}^{\prime}(\mathbf{0})\right\|, \\
\hat{\gamma} & :=\frac{\gamma}{1-q}\left\|\mathbf{V}^{T} \mathbf{F}^{\prime}(\mathbf{0})^{-1}\right\|\left\|\mathbf{V}^{T} \mathbf{F}^{\prime}(\mathbf{0})\right\|, \\
\kappa_{m}^{(1)} & :=\frac{\tau_{m} C_{D}}{(1-q) \hat{\alpha}}\left\|\mathbf{V}^{T} \mathbf{F}^{\prime}(\mathbf{0})^{-1}\right\|\left\|\mathbf{V}^{T}\right\|\left\|\left(\mathbf{I}_{N}-\mathbf{W} \mathbf{W}^{T}\right) \mathbf{f}\left(t_{m}, \mathbf{y}^{m}\right)\right\|, \\
\kappa_{m}^{(2)} & :=\frac{\gamma_{f} C_{D}}{(1-q) \hat{\gamma}}\left\|\mathbf{V}^{T} \mathbf{F}^{\prime}(\mathbf{0})^{-1}\right\|\left\|\mathbf{V}^{T}\right\|\left\|\left(\mathbf{I}_{N}-\mathbf{W} \mathbf{W}^{T}\right) \mathbf{f}_{\mathbf{y}}\left(t_{m}, \mathbf{y}^{m}\right)\right\| .
\end{aligned}
$$

Moreover, assume that

$$
\hat{h}_{m}:=\hat{\alpha}_{m} \hat{\gamma}_{m}<\frac{1}{2}, \quad \overline{\hat{B}}\left(\mathbf{0}, \hat{\rho}_{m}\right) \subset \hat{D}, \hat{\rho}_{m}:=\frac{1-\sqrt{1-2 \hat{h}_{m}}}{\hat{\gamma}_{m}} .
$$

Then, the sequence $\left\{\hat{\mathbf{x}}^{k}\right\}_{\mathbb{N}_{0}}$ of Newton iterates is well defined, stays in $\overline{\hat{B}}\left(\mathbf{0}, \hat{\rho}_{m}\right)$, and converges quadratically to $\hat{\mathbf{e}}^{m} \in \overline{\hat{B}}\left(\mathbf{0}, \hat{\rho}_{m}\right)$ with $\hat{\mathbf{F}}\left(\hat{\mathbf{e}}^{m}\right)=\mathbf{0}$. The solution $\hat{\mathbf{e}}^{m}$ is unique in $\overline{\hat{B}}\left(\mathbf{0}, \hat{\rho}_{m}\right) \cup\left(\hat{D} \cap \hat{B}\left(\mathbf{0}, \overline{\hat{\rho}}_{m}\right)\right), \overline{\hat{\rho}}_{m}:=\left(1+\sqrt{1-2 \hat{h}_{m}}\right) / \hat{\gamma}_{m}$.

Proof. Under assumption (4.49) the Jacobian $\hat{\mathbf{F}}^{\prime}(\mathbf{0})$ is regular and we obtain $\hat{\mathbf{F}}^{\prime}(\mathbf{0})^{-1} \hat{\mathbf{F}}(\mathbf{0})=$

$\tau_{m} \hat{\mathbf{F}}^{\prime}(\mathbf{0})^{-1} \mathbf{V}^{T}\left(\mathbf{I}_{N}-\mathbf{W}\left(\mathbf{P}^{T} \mathbf{W}\right)^{-1} \mathbf{P}^{T}\right) \mathbf{f}\left(t_{m}, \mathbf{y}^{m}\right) \mathbf{V}-\tau_{m} \hat{\mathbf{F}}^{\prime}(\mathbf{0})^{-1} \mathbf{V}^{T} \mathbf{f}\left(t_{m}, \mathbf{y}^{m}\right) \mathbf{V}=$ $\hat{\mathbf{F}}^{\prime}(\mathbf{0})^{-1} \mathbf{V}^{T} \mathbf{F}^{\prime}(\mathbf{0}) \mathbf{F}^{\prime}(\mathbf{0})^{-1} \mathbf{F}(\mathbf{0}) \mathbf{V}+\tau_{m} \hat{\mathbf{F}}^{\prime}(\mathbf{0})^{-1} \mathbf{V}^{T}\left(\mathbf{I}_{N}-\mathbf{W}\left(\mathbf{P}^{T} \mathbf{W}\right)^{-1} \mathbf{P}^{T}\right) \mathbf{f}\left(t_{m}, \mathbf{y}^{m}\right) \mathbf{V}$

For the first term on the right-hand side we get

$$
\left\|\hat{\mathbf{F}}^{\prime}(\mathbf{0})^{-1} \mathbf{V}^{T} \mathbf{F}^{\prime}(\mathbf{0}) \mathbf{F}^{\prime}(\mathbf{0})^{-1} \mathbf{F}(\mathbf{0}) \mathbf{V}\right\| \leq \frac{1}{1-q}\left\|\mathbf{V}^{T} \mathbf{F}^{\prime}(\mathbf{0})^{-1}\right\|\left\|\mathbf{V}^{T} \mathbf{F}^{\prime}(\mathbf{0})\right\|
$$

whereas the second term can be estimated from above as follows

$$
\begin{aligned}
& \tau_{m}\left\|\hat{\mathbf{F}}^{\prime}(\mathbf{0})^{-1} \mathbf{V}^{T}\left(\mathbf{I}_{N}-\mathbf{W}\left(\mathbf{P}^{T} \mathbf{W}\right)^{-1} \mathbf{P}^{T}\right) \mathbf{f}\left(t_{m}, \mathbf{y}^{m}\right) \mathbf{V}\right\| \leq \\
& \frac{\tau_{m} C_{D}}{1-q}\left\|\mathbf{V}^{T} \mathbf{F}^{\prime}(\mathbf{0})^{-1}\right\|\left\|\mathbf{V}^{T} \mathbf{F}^{\prime}(\mathbf{0})\right\|^{-1}\left\|\mathbf{V}^{T}\right\|\left\|\left(\mathbf{I}_{N}-\mathbf{W} \mathbf{W}^{T}\right) \mathbf{f}\left(t_{m}, \mathbf{y}^{m}\right)\right\| .
\end{aligned}
$$


Then, (4.58a) is a consequence of the preceding two estimates. In order to prove $(4.58 \mathrm{~b})$ we note that

$$
\begin{aligned}
& \hat{\mathbf{F}}^{\prime}(\mathbf{0})^{-1}\left(\mathbf{F}^{\prime}\left(\hat{\mathbf{y}}_{1}\right)-\mathbf{F}^{\prime}\left(\hat{\mathbf{y}}_{2}\right)\right)= \\
& \tau_{m} \hat{\mathbf{F}}^{\prime}(\mathbf{0})^{-1} \mathbf{V}^{T} \mathbf{F}^{\prime}(\mathbf{0}) \mathbf{F}^{\prime}(\mathbf{0})^{-1}\left(\mathbf{f}_{\mathbf{y}}\left(t_{m}, \mathbf{y}^{m}-\mathbf{V} \hat{\mathbf{y}}_{1}\right)-\mathbf{f}_{\mathbf{y}}\left(t_{m}, \mathbf{y}^{m}-\mathbf{V} \hat{\mathbf{y}}_{2}\right)\right) \mathbf{V}- \\
& \tau_{m} \hat{\mathbf{F}}^{\prime}(\mathbf{0})^{-1} \mathbf{V}^{T}\left(\mathbf{I}_{N}-\mathbf{W}\left(\mathbf{P}^{T} \mathbf{W}\right)^{-1} \mathbf{P}^{T}\right)\left(\mathbf{f}_{\mathbf{y}}\left(t_{m}, \mathbf{y}^{m}-\mathbf{V} \hat{\mathbf{y}}_{1}\right)-\mathbf{f}_{\mathbf{y}}\left(t_{m}, \mathbf{y}^{m}-\mathbf{V} \hat{\mathbf{y}}_{2}\right)\right) \mathbf{V}= \\
& \tau_{m} \hat{\mathbf{F}}^{\prime}(\mathbf{0})^{-1} \mathbf{V}^{T} \mathbf{F}^{\prime}(\mathbf{0}) \mathbf{F}^{\prime}(\mathbf{0})^{-1}\left(\mathbf{F}^{\prime}\left(\mathbf{V}_{\mathbf{y}}\right)-\mathbf{F}^{\prime}\left(\mathbf{V} \hat{\mathbf{y}}_{2}\right)\right) \mathbf{V}- \\
& \tau_{m} \hat{\mathbf{F}}^{\prime}(\mathbf{0})^{-1} \mathbf{V}^{T}\left(\mathbf{I}_{N}-\mathbf{W}\left(\mathbf{P}^{T} \mathbf{W}\right)^{-1} \mathbf{P}^{T}\right) \mathbf{f}_{\mathbf{y}}\left(t_{m}, \mathbf{y}^{m}\right) \mathbf{f}_{\mathbf{y}}\left(t_{m}, \mathbf{y}^{m}\right)^{-1} . \\
& \cdot\left(\mathbf{f}_{\mathbf{y}}\left(t_{m}, \mathbf{y}^{m}-\mathbf{V} \hat{\mathbf{y}}_{1}\right)-\mathbf{f}_{\mathbf{y}}\left(t_{m}, \mathbf{y}^{m}-\mathbf{V} \hat{\mathbf{y}}_{2}\right)\right) \mathbf{V} .
\end{aligned}
$$

The first term on the right-hand side can be estimated from above according to

$$
\begin{aligned}
& \tau_{m}\left\|\hat{\mathbf{F}}^{\prime}(\mathbf{0})^{-1} \mathbf{V}^{T} \mathbf{F}^{\prime}(\mathbf{0}) \mathbf{F}^{\prime}(\mathbf{0})^{-1}\left(\mathbf{F}^{\prime}\left(\mathbf{V} \hat{\mathbf{y}}_{1}\right)-\mathbf{F}^{\prime}\left(\mathbf{V} \hat{\mathbf{y}}_{2}\right)\right) \mathbf{V}\right\| \leq \\
& \frac{\gamma}{1-q} \tau_{m}\left\|\mathbf{V}^{T} \mathbf{F}^{\prime}(\mathbf{0})^{-1}\right\|\left\|\mathbf{V}^{T} \mathbf{F}^{\prime}(\mathbf{0})\right\|\left\|\hat{\mathbf{y}}_{1}-\hat{\mathbf{y}}_{2}\right\| .
\end{aligned}
$$

For the second term we obtain

$$
\begin{aligned}
& \tau_{m} \| \hat{\mathbf{F}}^{\prime}(\mathbf{0})^{-1} \mathbf{V}^{T}\left(\mathbf{I}_{N}-\mathbf{W}\left(\mathbf{P}^{T} \mathbf{W}\right)^{-1} \mathbf{P}^{T}\right) \mathbf{f}_{\mathbf{y}}\left(t_{m}, \mathbf{y}^{m}\right) \mathbf{f}_{\mathbf{y}}\left(t_{m}, \mathbf{y}^{m}\right)^{-1}\left(\mathbf{f}_{\mathbf{y}}\left(t_{m}, \mathbf{y}^{m}-\mathbf{V} \hat{\mathbf{y}}_{1}\right)-\right. \\
& \left.\mathbf{f}_{\mathbf{y}}\left(t_{m}, \mathbf{y}^{m}-\mathbf{V} \hat{\mathbf{y}}_{2}\right)\right) \mathbf{V}\left\|\leq \frac{C_{D} \gamma_{f}}{1-q} \tau_{m}\right\| \mathbf{V}^{T} \mathbf{F}^{\prime}(\mathbf{0})^{-1}\|\| \mathbf{V}^{T}\|\| \hat{\mathbf{y}}_{1}-\hat{\mathbf{y}}_{2} \| .
\end{aligned}
$$

The preceding two estimates result in $(4.58 \mathrm{~b})$. The rest of the assertions follow from Theorem 4.1.

REMARK 4.3. Let $\hat{\rho}:=(1-\sqrt{1-2 \hat{\alpha} \hat{\gamma}}) / \hat{\gamma}$ for $\hat{\alpha} \hat{\gamma}<1 / 2$ with $\hat{\alpha}, \hat{\gamma}$ as in (4.58e), (4.58f) and let $\hat{\rho}_{m}, 1 \leq m \leq M$, be given by (4.59) Then, with $\kappa_{m}^{(1)}$ as in (4.58g) there holds

$$
\frac{1}{2} \hat{\rho}\left(1+\kappa_{m}^{(1)}\right) \leq \hat{\rho}_{m} \leq 2 \hat{\rho}\left(1+\kappa_{m}^{(1)}\right)
$$

We know from [8] that there exists a constant $C>0$ such that

$$
\begin{aligned}
& \left\|\mathbf{e}^{m}-\mathbf{V} \hat{\mathbf{e}}^{m}\right\|=\| \mathbf{y}^{m}-\mathbf{y}_{T}^{m}-\left(\mathbf{V} \hat{\mathbf{y}}^{m}-\mathbf{V} \hat{\mathbf{y}}_{T}^{m} \| \leq\right. \\
& \left\|\mathbf{y}^{m}-\mathbf{V} \hat{\mathbf{y}}^{m}\right\|+\left\|\mathbf{y}_{T}^{m}-\mathbf{V} \hat{\mathbf{y}}_{T}^{m}\right\| \leq C \tau_{\text {DEIM }}, \quad \tau_{\text {DEIM }}:=\left(\sum_{\ell=n+1}^{N} \lambda_{\ell}+\sum_{\ell=L+1}^{N} s_{\ell}\right)^{1 / 2} .
\end{aligned}
$$

Again, we set $\hat{\eta}_{m}:=\left\|\hat{\mathbf{e}}^{m}\right\|, 1 \leq m \leq M$, and combine the results of Theorem (4.8) and (4.61) to prove error equilibration of the POD-DEIM based ROM.

THEOREM 4.9. In addition to the assumptions of Theorem 4.8 suppose that $\delta_{m}:=C \tau_{D E I M} / \eta_{m} \ll 1,1 \leq m \leq M$. Then, for $\eta_{m}=\eta\left(1-\varepsilon_{m}\right), \varepsilon_{m} \ll 1$, and $\Lambda:=\max (\hat{\rho}, \rho)$ it holds

$$
\Lambda^{-1} \frac{\left(1-\varepsilon_{m}\right)\left(1-\delta_{m}\right)}{2\left(1+\kappa_{m-1}^{(1)}\right)} \leq \frac{\hat{\eta}_{m}}{\hat{\eta}_{m-1}} \leq \Lambda \frac{2\left(1+\kappa_{m}^{(1)}\right)}{\left(1-\delta_{m-1}\right)\left(1-\varepsilon_{m-1}\right)}
$$

Proof. In much the same way as in the proof of Theorem 4.7 we derive

$$
\frac{\eta_{m}\left(1-C \tau_{\text {DEIM }} / \eta_{m}\right)}{\max (\hat{\rho}, \rho)} \leq \frac{\hat{\eta}_{m}}{\hat{\eta}_{m-1}} \leq \frac{\max (\hat{\rho}, \rho)}{\eta_{m-1}\left(1-C \tau_{\text {DEIM }} / \eta_{m-1}\right)},
$$

which gives (4.62) by taking (4.60) into account. 
5. Numerical results. As an example for a semilinear parabolic initial-boundary value problem we consider

$$
\begin{array}{rlrl}
\frac{\partial y}{\partial t}-\Delta y+y^{2} & =f & & \text { in } Q \\
y=0 & & \text { on } \Sigma, \\
y(\cdot, 0)=0 & & \text { in } \Omega,
\end{array}
$$

where $Q:=\Omega \times(0,1), \Omega:=(0,1), \Sigma:=\Gamma \times(0,1), \Gamma:=\partial \Omega$, and the right-hand side $f$ is given such that

$$
y(x, t)=x^{3}(1-x)^{3} t^{2}(1-t)^{2} \arctan \left(60\left(\left(x-\frac{5}{4}\right)^{2}+\left(t+\frac{1}{4}\right)^{2}\right)^{1 / 2}-1\right)
$$

is the exact solution of (5.1a)-(5.1c) (cf. Figure 5.1).

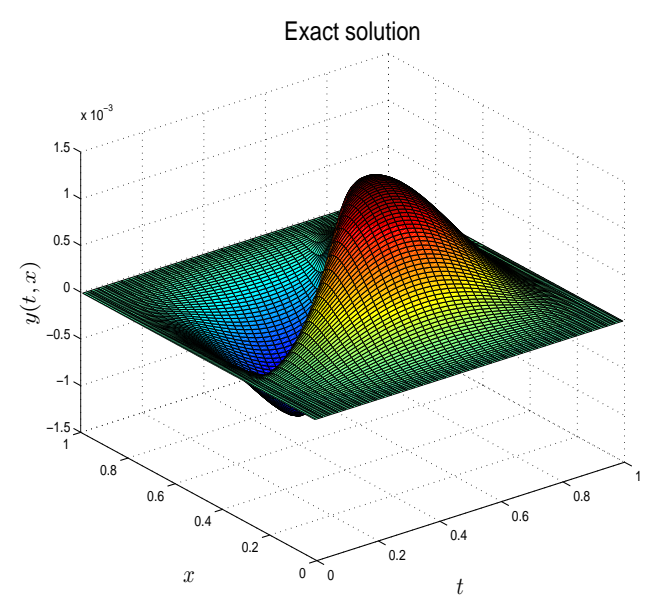

FIG. 5.1. Exact solution of the semilinear parabolic PDE.

As the nonlinear FOM we have chosen a finite element approximation in space by continuous, piecewise linear finite elements with respect to a uniform partition of the computational domain $\Omega$ with mesh size $h=1 / 128$. We have further chosen 42 snapshot locations in the time interval $(0,1)$ by the solution of the minimization problem (4.8) of section 4 .

We have used the implicit Euler finite element solutions to compute both the POD and the POD-DEIM singular values and basis functions. The singular values of the POD snapshot matrix and the POD-DEIM snapshot matrix are displayed in Figure 5.3 and Figure 5.4.

Table 1 contains the averages of the error estimators $\eta_{m}$ and $\hat{\eta}_{m}$ and its standard deviations for the FOM and the POD and POD-DEIM ROMs of different dimension $N$. The results reflect the theoretically derived estimates of subsection 4.3 
Plot of 40 time instances for error equilibration

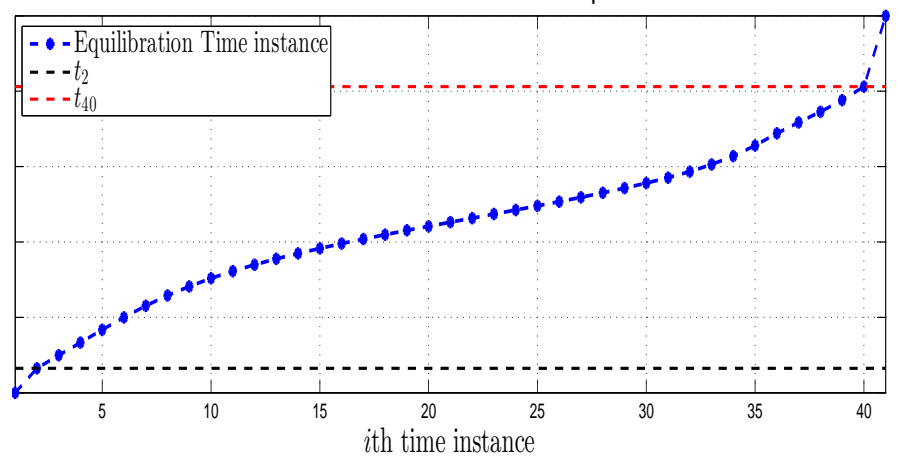

FIG. 5.2. Snapshot locations $t_{m}, 0 \leq m \leq 41$, by error equilibration for the FOM.

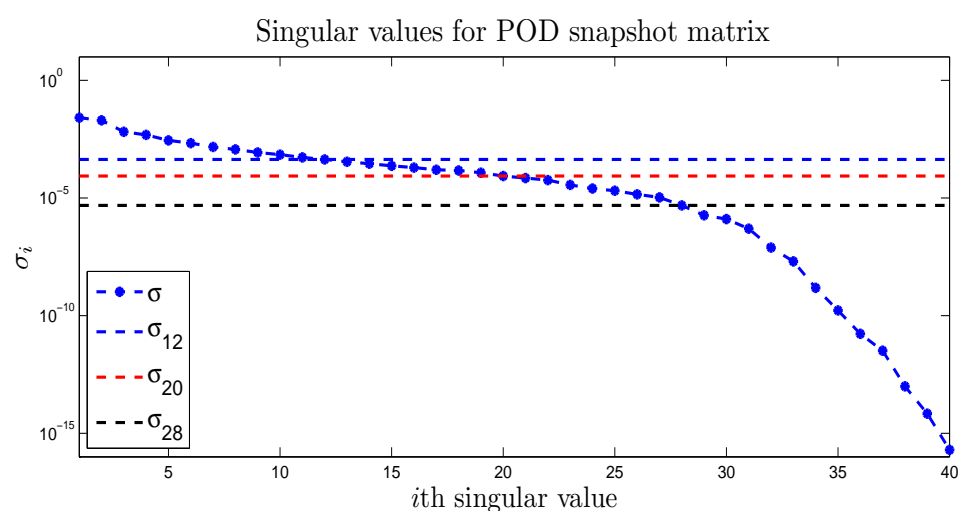

FIG. 5.3. Singular values of the POD snapshot matrix.

\section{REFERENCES}

[1] A. C. Antoulas; Approximation of Large-Scale Systems, SIAM, Philadelphia, 2005.

[2] M. Barrault, Y. Maday, N. C. Nguyen, and A. T. Patera; An 'empirical interpolation' method: application to efficient reduced-basis discretization of partial differential equations, Comptes Rendus Mathematique 339, 667-672, 2004.

[3] P. Benner, M. Hinze, and E.J.W. ter Maten (eds.); Model Reduction for Circuit Simulation, Springer, Berlin-Heidelberg-New York, 2011.

[4] P. Benner, V. Mehrmann, and D. C. Sorensen (eds.); Dimension Reduction of LargeScale Systems, Lecture Notes in Computational Science and Engineering, Vol. 45, Springer, Berlin-Heidelberg-New York, 2005.

[5] P.T. Boggs and J.W. Tolle; Sequential quadratic programming, Acta Numerica 4, $1-51,1995$.

[6] S.C. Brenner and L. Ridgway Scott, The Mathematical Theory of Finite Element Methods. 3rd Edition, Springer, New York, 2008

[7] S. Chaturantabut and D.C. Sorensen; Discrete empirical interpolation for nonlinear model reduction, SIAM J. Sci. Comput. 32, 2737-2764, 2010.

[8] S. Chaturantabut and D.C. Sorensen; A state space error estimate fpr POD-DEIM nonlinear model reduction, Technical Report TR10-32, CAAM, Rice University, Houston, 2010.

[9] Z. Chen and J. Feng; An adaptive finite element algorithm with reliable and efficient 


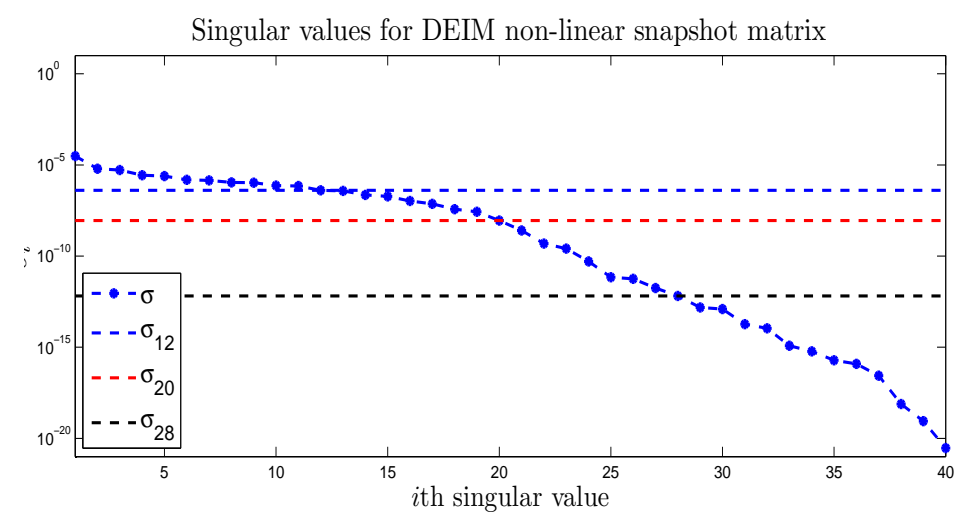

FIG. 5.4. Singular values of the POD-DEIM snapshot matrix.

TABLE 5.1

Average of the error estimators $\eta_{m}$ and $\hat{\eta}_{m}$ and its standard deviations for the FOM and the $P O D$ and POD-DEIM ROMs of different dimension $N$

\begin{tabular}{|c|c|c|c|}
\hline FOM & $N$ & POD & POD-DEIM \\
\hline $3.707 E-4 \pm 5.025 E-8$ & 12 & $3.629 E-4 \pm 1.064 E-4$ & $3.631 E-4 \pm 1.094 E-4$ \\
& 20 & $3.629 E-4 \pm 6.752 E-6$ & $3.642 E-4 \pm 6.906 E-5$ \\
& 28 & $3.695 E-4 \pm 5.964 E-7$ & $3.703 E-4 \pm 1.910 E-6$ \\
& 40 & $3.696 E-4 \pm 6.080 E-7$ & $3.696 E-4 \pm 6.080 E-7$ \\
\hline
\end{tabular}

error control for linear parabolic problems. Math. Comp. 73, 1167-1193, 2004.

[10] P.G. Ciarlet; The Finite Element Method for Elliptic Problems, SIAM, Philadelphia, 2002.

[11] P. Deuflhard; Newton Methods for Nonlinear Problems. Affine Invariance and Adaptive Algorithms, Springer, Berlin-Heidleberg-New York, 2004.

[12] P. Deuflhard anf F. Bornemann; Scientific Computing with Ordinary Differential Equations, Springer, Berlin-Heidelberg-New York, 2002.

[13] M. A. Grepl, Y. Maday, N. C. Nguyen, and A. T. Patera; Efficient reduced-basis treatment of nonaffine and nonlinear partial differential equations, Mathematical Modelling and Numerical Analysis 41, 575-605, 2007.

[14] S. Gugercin and A.C. Antoulas; A survey of model reduction by balanced truncation and some new results. Int. J. Control 77, 748-766, 2004.

[15] T. Henri and J.-P. Yvon; Convergence estimates of POD-Galerkin methods for parabolic problems. In: System Modeling and Optimization. IFIP Int. Fed. for Inf. Processing. Vol. 166, pages 295-306, Springer, Boston, 2005.

[16] D. Henry; Geometric Theory of Semilinear Parabolic Equations, Springer, BerlinHeidelberg-New York, 1981.

[17] C. Homescu, L. R. Petzold, and R. Serban; Error estimation for reduced-order models of dynamical systems, SIAM Review 49, 277-299, 2007.

[18] T. Kato; Nonlinear semigroups and evolution equations, J. Math. Soc. Japan 19, 503520, 1967.

[19] N. Kenmochi and S. Oharu; Difference approximation of nonlinear evolution equations and semigroups of nonlinear operators, Publ. RIMS, Kyoto Univ. 10, 147-207, 1974.

[20] K. Kunisch and S. Volkwein; Galerkin proper orthogonal decomposition methods for parabolic problems. Numer. Math. 90, 117-148, 2001.

[21] K. Kunisch and S. Volkwein; Galerkin proper orthogonal decomposition methods for a general equation in fluid dynamics. SIAM J. Numer. Anal. 40, 492-515, 2002. 
[22] K. Kunisch and S. Volkwein; Optimal snapshot location for computing POD basis functions, ESAIM: Mathematical Modelling and Numerical Analysis 44, 509-529, 2010 .

[23] O.A. Ladyzenskaja, V.A. Solonnikov, and N.N. Ural'ceva; Linear and Quasilinear Equations of Parabolic Type, AMS, Providence, 1968

[24] H.V. Ly and H.T. Tran; Modelling and control of physical processes using proper orthogonal decomposition. Math. and Comput. Modelling 33, 223-236, 2001.

[25] B. C. Moore; Principal component analysis in linear systems: controllability, observability, and model reduction, IEEE Trans. Automat. Control 26, 17-32, 1981.

[26] B. R. Noack, M. Morzynski, and G. Tadmor (eds.); Reduced-Order Modelling for Flow Control. Springer, Berlin-Heidelberg-New York, 2011.

[27] G. Obinata and B.D.O. Anderson; Model Reduction for Control System Design, Springer, Berlin-Heidelberg-New York, 2001.

[28] J.M. Ortega and W.C. Rheinboldt; Iterative Solution of Nonlinear Equations in Several Variables, SIAM, Philadelphia, 2000.

[29] M. Renardy and R.C. Rogers, An Introduction to Partial Differential Equations, Springer, Berlin-Heidelberg-New York, 1993.

[30] W.H. Schilders, H.A. van der Vorst, and J. Rommes (eds.); Model Order Reduction: Theory, Research Aspects, and Applications, Springer, Berlin-HeidelbergNew York, 2008.

[31] L. Tartar; Introduction to Sobolev Spaces and Interpolation Theory, Springer, BerlinHeidelberg-New York, 2007.

[32] V. Thomée; Galerkin Finite Element Methods for Parabolic Problems, Springer, Berlin-Heidelberg-New York, 1997.

[33] S. Volkwein; Model Reduction using Proper Orthogonal Decomposition, Lecture Notes, Institute of Mathematics and Scientific Computing, University of Graz, 2008 (see http://www.uni-graz.at/imawww/volkwein/POD.pdf).

[34] K. Zhou, J. C. Doyle, and K. Glover; Robust and Optimal Control, Prentice Hall, Englewood Cliffs, 1996. 